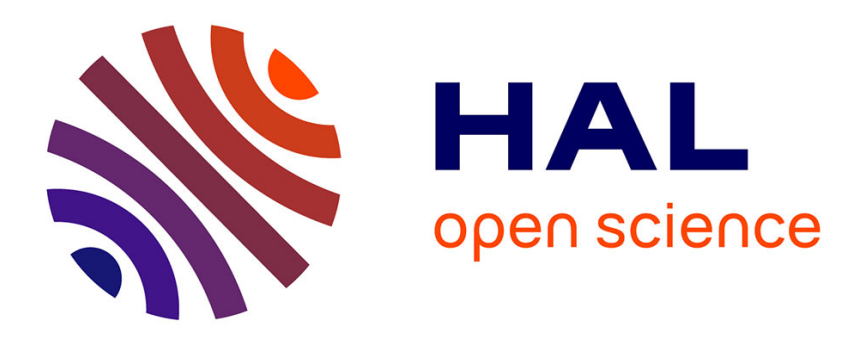

\title{
Effect of Perforated Plates on the Acoustics of Annular Combustors
}

\author{
Elsa Gullaud, Franck Nicoud
}

\section{To cite this version:}

Elsa Gullaud, Franck Nicoud. Effect of Perforated Plates on the Acoustics of Annular Combustors. AIAA Journal, 2012, 50 (12), pp.2629-2642. 10.2514/1.J050716 . hal-00800709

\section{HAL Id: hal-00800709 \\ https://hal.science/hal-00800709}

Submitted on 14 Mar 2013

HAL is a multi-disciplinary open access archive for the deposit and dissemination of scientific research documents, whether they are published or not. The documents may come from teaching and research institutions in France or abroad, or from public or private research centers.
L'archive ouverte pluridisciplinaire HAL, est destinée au dépôt et à la diffusion de documents scientifiques de niveau recherche, publiés ou non, émanant des établissements d'enseignement et de recherche français ou étrangers, des laboratoires publics ou privés. 


\title{
On the effect of perforated plates on the acoustics of
}

\section{annular combustors}

\author{
E. Gullaud ${ }^{1}$ \\ CERFACS, Toulouse, France \\ F. Nicoud ${ }^{2}$ \\ University Montpellier II, 34095 Montpellier, France
}

This paper aims at showing the influence of perforated plates on the acoustic modes in aeronautical gas turbines combustion chambers. The analytical model developed by Howe was implemented in a 3D acoustic Helmholtz solver to account for the effect of perforated plates. First, an analytic test case is used to validate the coding in the acoustic solver. Then, a computation of the acoustic modes in an actual industrial chamber is conducted, taking into account the perforated liners. For both cases, a study of the influence of the bias flow speed is conducted. The acoustic energy budget is also used to evaluate the respective contributions of the perforated plates. In the case of the industrial chamber, some plates are proved to be more effective than the others, depending on the mode structure.

\footnotetext{
1 PhD Student, CERFACS, 42, av. Gaspard Coriolis, gullaud@cerfacs.fr.

2 Professor, Université Montpellier II, I3M - CNRS UMR 5149 - CC51; franck.nicoud@univ-montp2.fr .
} 


\section{Nomenclature}

$N \quad=$ Number of dicretization nodes

A,B $=\mathrm{NxN}$ matrices

$\mathbf{P}=$ Acoustic pressure amplitude, $\mathrm{N}$ vector

$\hat{p} \quad=$ Spatial pressure fluctuation

$\hat{\mathbf{u}} \quad=$ Spatial velocity fluctuation

$\mathbf{n} \quad=$ Unit normal vector

$\Omega \quad=$ Computational domain

$\delta \Omega \quad=$ Boundary of the computational domain

$\delta \Omega_{Z} \quad=$ Boundary on which an impedance condition is applied

$\delta \Omega_{W}=$ Boundary on which an impermeable condition is applied

$\delta \Omega_{P} \quad=$ Boundary on which a null acoustic pressure is applied

$\delta \Omega_{M P}=$ Boundary on which a perforated plate condition is applied

$\gamma \quad=$ Polytropic coefficient

$\rho_{0} \quad=$ Density $k g \cdot m^{-3}$

$\nu \quad=$ Cinematic viscosity $m^{2} \cdot s^{-1}$

$p_{0} \quad=$ Mean pressure $P a$

$a \quad=$ Aperture radius $\mathrm{m}$

$c \quad=$ Sound speed $m \cdot s^{-1}$

$d \quad=$ Aperture spacing $\mathrm{m}$

$U \quad=$ Bias flow speed $m \cdot s^{-1}$

$U_{m d} \quad=$ Speed of maximal damping $m \cdot s^{-1}$

$f \quad=$ Mode frequency $\mathrm{Hz}$

$\omega \quad=$ Angular frequency, $r a d . s^{-1}$

$k=\frac{\omega}{c}=$ Wave number, rad. $m^{-1}$

$\omega_{r} \quad=$ Real part of $\omega$, rad.s $s^{-1}$

$\omega_{i} \quad=$ Imaginary part of $\omega$, rad.s $s^{-1}$

$S_{t} \quad=$ Strouhal number $\frac{\omega_{r} a}{U}$

$K_{a} \quad=$ Rayleigh conductivity $m^{-1}$

$t \quad=$ Time

$(r, \theta, z)=$ Polar coordinates 


\section{Conventions}

$\nabla z=$ Spatial gradient of $\mathrm{z}$

$i \quad=$ Imaginary complex number

$\operatorname{Im}()=$ Imaginary part

$R e()=$ Real part

()$^{*}=$ Complex conjugate

()$^{\prime}=$ Derivative

\section{Subscripts}

$0=$ time index for averaged quantities

\section{Superscripts}

$+=$ value upstream

- = value downstream

$1=$ Fluctuating quantity

\section{Introduction}

In order to cut down pollutant emissions, industrial gas turbine combustion chambers are run in the lean premixed regime which on the other hand promotes combustion instabilities [1, 2]. These instabilities result from a constructive coupling between the combustion process and the acoustic modes of the chamber. They might cause harmful damages to the combustion chamber and therefore need to be accurately predicted.

Massive parallel computations and Large Eddy Simulations (LES) make now possible to represent the complex turbulent flow within combustion chambers [3-5]. Such LES solvers can predict instabilities in a reacting flow configuration $[2,6]$ but their cost for full annular chambers remains prohibitive [7, 8]. Moreover, these simulations cannot be repeated easily and can not be used to optimize chamber designs to control azimuthal modes. 
But in the cases where the flame response is known [9, 10], acoustic Helmholtz solvers can prove to be sufficient to compute the thermoacoustic modes of a combustor. Helmholtz solvers reduce cost compared to solving the full Navier-Stokes equations [11-13]. To improve accuracy of these computations, the whole complex geometry of the combustor, including the combustion chamber, casing, swirlers and dilution holes need to be taken into account $[14,15]$.

Walls of recent combustion chambers in aeronautical gas turbines are generally perforated with sub-millimeter holes for cooling purposes. These holes allow fresh air flowing in the casing (outside the combustion chamber) to enter the combustion chamber due to the pressure difference across the walls. The jets issuing from the holes coalesce to form a protecting cooling film that isolates the wall from the hot gases flowing in the chamber. This technique is called full-coverage film cooling (FCFC) [16].

In addition to modifying the dynamical and the thermal characteristics of the flow near the walls, FCFC may have an impact on the acoustics of the chamber. Indeed, multiperforated plates (MP) are known to have a damping effect on incident acoustic waves [17-21]. Acoustic waves force the shear layer between the jet and the main flow to break down and form vortex rings. This mechanism efficiently converts part of the acoustic energy into vortical energy [22]. Vortices are in turn dissipated by viscosity without substantial sound production [23]. The damping effect is generally enhanced by the presence of a bias flow through the plate [23-25], as encountered in FCFC. It stronly depends on the parameters of this bias flow [24], on the geometrical characteristics of the plate (porosity or shape of the hole) [26, 27] and on the amplitude of the perturbation [28, 29].

To perform predictive simulations of combustion instabilities, one should then either account for the presence of multiperforated walls in the chamber, or show that their effect can be neglected. To the authors' knowledge, no study in the literature has properly addressed this question. Indeed, accounting for FCFC in simulations is a difficult task: due to the tiny diameter of the apertures, meshing the holes in numerical computations is clearly impossible. It is thus necessary to use models to represent the macroscopic effect of perforated walls on both near wall flow structure and acoustics. This issue is encountered in computational fluid dynamics calculations [30-32], but also when resolving the Helmholtz equation to determine the acoustic modes of a chamber. 
Analytical models have been developed and can be used to model the acoustic behaviour of MP. Howe proposed a model to represent the acoustic damping due to the vortex shedding in perforated plates submitted to a bias flow. Howe's model [23] provides the acoustic impedance of a MP and is therefore well adapted to Helmholtz solvers. To account for the plate thickness, which is neglected in Howe's model, Jing and Sun [33] proposed a modified version of Howe's model. However, the use of the modified model does not provide significant difference on the configurations studied here. Melling's model [21] also provides the impedance of a MP considering the damping is due to the shear layers inside the orifices. Efforts have also been made to account for the presence of the grazing flow $[34,35]$, but no analytical model has yet been developed and thus cannot be implemented numerically.

In the present study, we will show how such models can be integrated in a general purpose Helmholtz solver to account for the presence of perforated walls in aeronautical combustion chambers. The resulting tool will then be applied to a helicopter industrial chamber. The derivation of the Helmholtz equation under low Mach assumption is recalled in section II A and the Helmholtz solver is described in section II B. A short overview of the available analytical models for MP will be given in II C and their numerical implementation is explained in IID. Section III is dedicated to the verification of the implementation of model derived by Howe in an academic test case consisting in two coaxial cylinders separated by a perforated plate. A study of the influence of the parameters is also conducted in this section. Section IV shows how an energy budget can be computed from the results of the Helmholtz solver. Computations and energy budgets are performed on the longitudinal modes of a real industrial chamber in Section V. Finally, a computation on the whole chamber allows to obtain the azimuthal modes of the industrial chamber in Section VI.

\section{Incorporating MP models into a Helmholtz solver}

\section{A. Derivation of the Helmholtz equation under low Mach assumption}

Under the hypothesis of a non viscous, low Mach number mean flow and linear acoustics, the wave equation can be written in a non reactive media as follows [2]:

$$
\frac{1}{\gamma p_{0}} \frac{\partial^{2} p_{1}}{\partial t^{2}}-\nabla \cdot\left(\frac{1}{\rho_{0}} \nabla p_{1}\right)=0
$$


Harmonic variations are supposed for $p_{1}$ and $\mathbf{u}_{1}$, such that $\mathbf{u}_{1}=\operatorname{Re}\left(\hat{\mathbf{u}}(\mathbf{x}) e^{-i \omega t}\right)$ and $p_{1}=$ $\operatorname{Re}\left(\hat{p}(\mathbf{x}) e^{-i \omega t}\right)$. In the frequency domain, the wave equation becomes the Helmholtz equation

$$
\nabla \cdot c^{2} \nabla \hat{p}+\omega^{2} \hat{p}=0
$$

which is valid over the interior of the flow domain $\Omega$.

Boundary conditions are formally written as a Robin condition:

$$
c Z \nabla \hat{p} \cdot \mathbf{n}-i \omega \hat{p}=0
$$

where $\mathbf{n}$ is the unit vector normal to the domain boundary $\delta \Omega$ and $Z$ is the complex impedance on $\delta \Omega$.

\section{B. Description of the Helmholtz solver used in this study}

The Helmholtz solver used in this study solves the Helmholtz equation (Eq. (2)) and is fully described in [36]. A finite element method allows to turn Eq. (2) into the following matricial form:

$$
\mathbf{A P}+\omega \mathbf{B P}+\omega^{2} \mathbf{P}=0
$$

where $\mathbf{A}$ is a matrix of size $N \times N$ corresponding to the operator $\nabla \cdot c^{2} \nabla \hat{p}, \mathbf{B}$ is the matrix corresponding to the boundary conditions and $\mathbf{P}$ is the column vector of size $N$ giving the nodal values of pressure. The size $N$ of the problem is the number of nodes in the finite element grid that discretizes the geometrical domain. The eigen value problem of Eq. (4) is solved numerically using a parallel implementation of the Arnoldi method available in the P-ARPACK library [37]. The dependence in $\omega$ of $\mathrm{Z}$ implies that the matrix $\mathbf{B}$ is a non linear function of the frequency. The non-linearity of the problem is handled by an iterative method; each sub iteration considers that the matrix $\mathbf{B}$ is constant and resolves a linear or quadratic eigenvalue problem [36]. The eigenelements of Eq. (4) thus provide the field of harmonic acoustic pressure and the angular frequency.

\section{Acoustic models for MP}

Let us consider an array of circular apertures of radius $a$, with an aperture spacing $d$, through which a mean flow parallel to the apertures axis flows at speed $U$. Under the excitation of an acoustic 
wave, Howe's model allows representing the response of this flow under the following hypotheses [23]:

- The acoustic excitation is at a low frequency, so that the wavelength is much larger than the orifices radius,

- the flow has a high Reynolds number, the viscosity is then only dominant at the rims of the aperture leading to the shedding of vortices,

- the Mach number of the mean flow is low, so that the flow is incompressible in the vicinity of the aperture,

- the plate is infinitely thin,

- the aperture spacing is high compared to the aperture radius, so that the interaction between the apertures is negligible and single-aperture results can be applied to the MP plate.

The reasoning is then done for an isolated hole. The back plane shown on Fig. 1 is located at a distance a much higher than the aperture radius, thus being considered infinitely far away, making possible the application of Howe's model.

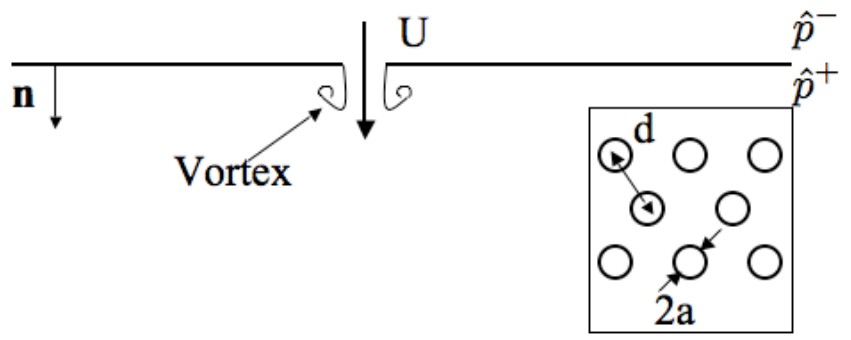

Fig. 1 Array of circular apertures, of diameter 2a and aperture spacing d, with a bias flow of speed U.

The Rayleigh conductivity $K_{a}[38]$ of the aperture, relating the harmonic volumic flux $\hat{Q}$ to the acoustic pressure jump across the plate, is defined by

$$
K_{a}=\frac{i \omega \rho_{0} \hat{Q}}{\hat{p}^{+}-\hat{p}^{-}}
$$


where $\rho_{0}$ is the mean density in the vicinity of the aperture, $\omega$ is the pulsation of the acoustic perturbation and $\hat{p}^{-}$and $\hat{p}^{+}$are the harmonic pressures upstream and downstream of the aperture respectively. We have

$$
\hat{Q}=d^{2} \hat{u}^{ \pm}
$$

where $\hat{u}^{ \pm}$is the acoustic velocity on the plate, equal on both sides. Hence,

$$
K_{a}=\frac{i \omega \rho_{0} d^{2} \hat{u}^{ \pm}}{\hat{p}^{+}-\hat{p}^{-}}
$$

Howe expresses the Rayleigh conductivity for a circular aperture in an infinitely thin plate as:

$$
K_{a}=2 a\left(\Gamma_{a}-i \Delta_{a}\right)
$$

where

$$
\Gamma_{a}-i \Delta_{a}=1+\frac{\frac{\pi}{2} I_{1}(S t) e^{-S t}-i K_{1}(S t) \sinh (S t)}{S t\left(\frac{\pi}{2} I_{1}(S t) e^{-S t}+i K_{1}(S t) \cosh (S t)\right)}
$$

with $S t=\frac{\omega_{r} a}{U}$ and $i^{2}=-1$.

The expression of the Rayleigh conductivity has been modified by Jing and Sun [34] to take into account the thickness $h$ of the plate:

$$
K_{a}=2 a\left(\frac{1}{\Gamma_{a}-i \Delta_{a}}+\frac{2 h}{\pi a}\right)^{-1}
$$

Luong et al. [39] proposed a simplified expression of the Rayleigh conductivity

$$
K_{a}=2 a\left(\frac{S_{t}}{S_{t}+\frac{2 i}{\sigma_{j}}}\right),
$$

where $\sigma_{j}$ is the contraction ratio of the radius of the jet, due to the vena contracta effect [17]. The value of the Rayleigh conductivity derived by Luong et al. is close to the one of Howe in the case of a contraction ratio of $\sigma_{j}=0.75$.

Another analytic approach was used by Melling [21] to derive the Rayleigh conductivity. This approach is based on the linearization of the Navier-Stokes equations inside each orifice. The dissipation of sound is supposed in this model to be due to viscous shear layers instead of the vortex 
shedding as assumed by Howe. Considering $l_{\nu}=\sqrt{\frac{2 \nu}{\omega}}$ the thickness of the viscous shear layer the following expression for the Rayleigh conductivity is derived:

$$
K_{a}=\frac{\pi a^{2}}{h\left(1+\frac{l_{\nu}}{a}(1+i)\right)} .
$$

Assuming that the distance between the apertures is large enough $(d \gg a)$, the interactions between the holes can be neglected. Then, the above expressions for $K_{a}$ can be used to describe the acoustic effect of a MP submitted to an acoustic perturbation whose wave length is large compared to $d$. Besides, all the models recalled above can be seen as Eq. (7) complemented by an expression for the Rayleigh conductivity of the form

$$
K_{a}=K_{a}\left(a, h, \sigma_{j}, S_{t}\right)
$$

where the first two parameters describe the aperture and the last two describe the mean and unsteady components of the bias flow. In the following, we will simply refer to the Rayleigh conductivity as $K_{a}$ so that the numerical implementation remains valid for any of the above acoustic models for MP.

\section{Numerical implementation}

Using the momentum equation and Eq. (7), the following relation is obtained between the acoustic pressure jump across the plate and the acoustic pressure gradient:

$$
\boldsymbol{\nabla} \hat{p} \cdot \mathbf{n}=\frac{K_{a}}{d^{2}}\left[\hat{p}^{+}-\hat{p}^{-}\right]
$$

The numerical implementation of a MP model is first discussed in the isothermal case where the mean density can be considered the same on both sides of the plate. Figure 2 illustrates the implementation of any acoustic model of the form of Eq. (7) in a Helmholtz solver. The basic idea is to build a boundary condition imposed on each side of the homogeneous MP in such a way to retrieve Eq. (7) when computing the eigenfrequencies. Note that since the actual MP has a finite thickness, the two sides of the plate are not located at the same place. Of course, no acoustic 


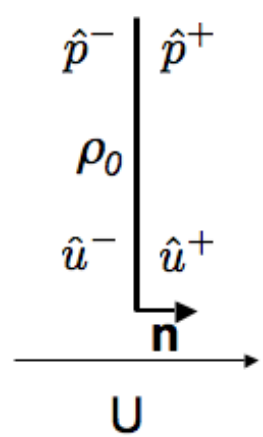

Schematic formulation of an acoustic model.

Plate may be infinitely thin

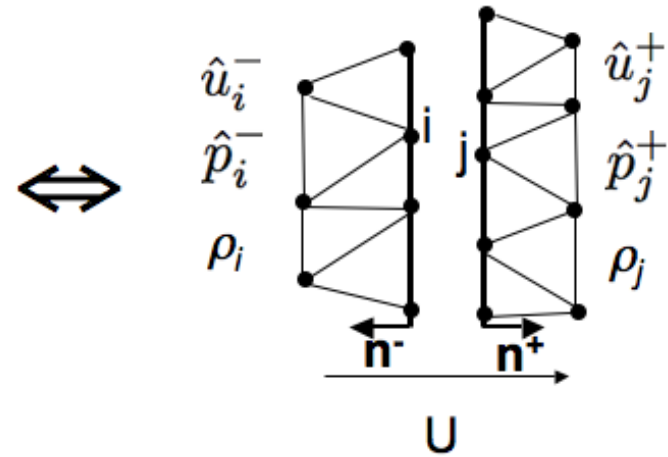

Situation in the solver:

Discretization of the fields

of $\hat{p}, \hat{\mathbf{u}} . \rho_{0}=\rho_{j}=\rho_{i}$.

Fig. 2 Equivalence between the Howe model and the discretized expression in the Helmholtz solver. Isothermal case.

pressure is defined within the homogenized MP and the region between the two sides is not meshed. Let us assume that each side of the MP is oriented such that the local normal vector is pointing towards the flow domain (see $\mathbf{n}^{+}$and $\mathbf{n}^{-}$in Fig. 2). The momentum equation under the zero Mach number assumption reads

$$
i \omega \rho_{0} \hat{\mathbf{u}} \cdot \mathbf{n}^{ \pm}=\nabla \hat{p} \cdot \mathbf{n}^{ \pm}
$$

and the continuity of the acoustic velocity leads to

$$
\boldsymbol{\nabla} \hat{p} \cdot \mathbf{n}^{+}=-\nabla \hat{p} \cdot \mathbf{n}^{-}
$$

Eq. (7) then leads to

$$
\left\{\begin{array}{cc}
i \omega \rho_{0} \hat{\mathbf{u}} \cdot \mathbf{n}^{+}=\frac{K_{a}}{d^{2}}\left[\hat{p}^{+}-\hat{p}^{-}\right] & \text {on the downstream side. } \\
i \omega \rho_{0} \hat{\mathbf{u}} \cdot \mathbf{n}^{-}=\frac{K_{a}}{d^{2}}\left[\hat{p}^{-}-\hat{p}^{+}\right] & \text {on the upstream side. }
\end{array}\right\}
$$

Finally, the acoustic model of the MP leads to the following condition valid on each side of the plate:

$$
\nabla \hat{p} \cdot \mathbf{n}=-\frac{K_{a}}{d^{2}}[\hat{p}]
$$


where $[\hat{p}]$ is the acoustic pressure jump defined as the value of $\hat{p}$ on the opposite side minus the value of the current node.

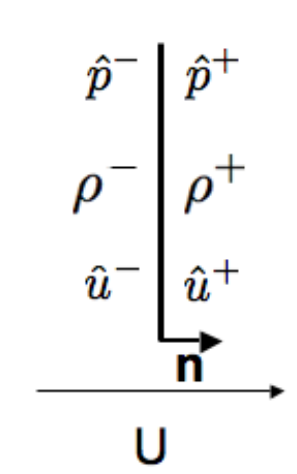

Schematic formulation of an acoustic model. Plate may be infinitely thin

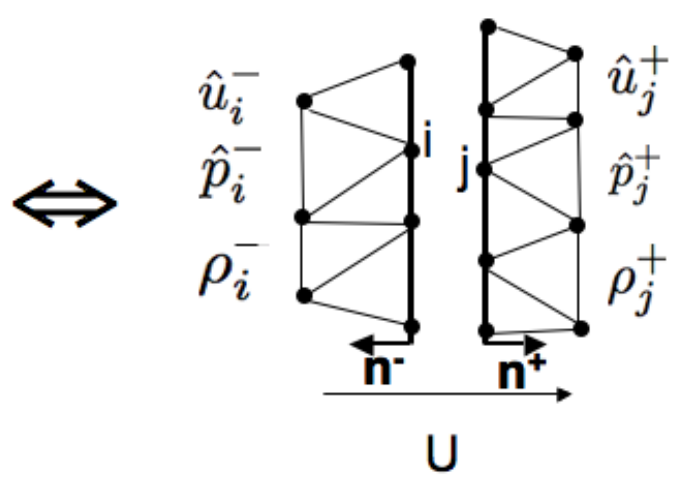

Situation in the solver:

Discretization of the fields

of $\hat{p}, \hat{\mathbf{u}}$ and $\rho$.

Fig. 3 Equivalence between the Howe model and the discretized expression in the Helmholtz solver. Anisothermal case.

In the case of a combustion chamber, the upstream flow coming from the casing of the chamber is cold (of volumic mass $\rho^{-}$), and the downstream flow located in the combustion chamber is hot (with a volumic mass $\rho^{+}$). The equality of acoustic speeds through the plate then reads:

$$
\frac{1}{\rho^{+}} \boldsymbol{\nabla} \hat{p} \cdot \mathbf{n}^{+}=\frac{-1}{\rho^{-}} \nabla \hat{p} \cdot \mathbf{n}^{-}
$$

Considering that the density to be used in Eq. (7) is the density of the upstream gas, the condition to implement on the upstream side of the plate remains the same as for the isothermal case:

$$
\boldsymbol{\nabla} \hat{p} \cdot \mathbf{n}^{-}=-\frac{K_{a}}{d^{2}}\left[\hat{p}^{+}-\hat{p}^{-}\right]
$$

In order to comply with the continuity of the acoustic velocity, it is then necessary to impose

$$
\boldsymbol{\nabla} \hat{p} \cdot \mathbf{n}^{+}=-\frac{\rho^{+}}{\rho^{-}} \frac{K_{a}}{d^{2}}\left[\hat{p}^{-}-\hat{p}^{+}\right]
$$


on the downstream side of the plate. The generic condition to implement for any thermal condition on a node $j$ is then:

$$
\boldsymbol{\nabla} \hat{p} \cdot \mathbf{n}_{j}=-\frac{\rho_{j}}{\rho^{-}} \frac{K_{a}}{d^{2}}[\hat{p}]^{j},
$$

where $\rho_{j}$ is the mean density at node $j, \mathbf{n}_{j}$ is the inward normal vector defined at node $j$ and $[\hat{p}]^{j}$ is the pressure jump at node $j$. Note that in most of the cases, an interpolation procedure is necessary to get the corresponding pressure on both sides and build the appropriate pressure jump.

Eq. (22) can then be used as a Neumann boundary condition in the Helmholtz solver. The normal pressure gradient on each multiperforated boundary is expressed as a function of $a, d, U$ and $\omega$ so that the numerical treatment is similar to what is done for the finite impedance boundaries. Formaly, this amounts to impose

$$
Z^{j}=\frac{i \omega \hat{p}_{j}}{c_{0} \boldsymbol{\nabla} \hat{p} \cdot \mathbf{n}_{j}}=-\frac{i \omega \rho^{-} d^{2} \hat{p}_{j}}{c_{0} \rho_{j} K_{a}[\hat{p}]^{j}}
$$

on both sides of the MP. The corresponding terms are then included into the matrix $\mathbf{B}$ of Eq. (4).

\section{Analytic validation of the coding}

First, an academic configuration for which an analytic solution can be derived is presented. Some of the material presented in this section was already given in [40] and is recalled here for completeness.

The geometry depicted in Fig. 4 is computed both numerically and analytically. It consists of two coaxial cylinders, the inner one being perforated. The outer boundary of radius $r_{2}$ consists of a hard wall and will be noted $\delta \Omega_{W}$. The perforated plate is located at $r=r_{1}$. The boundary denoted by the MP will be noted $\delta \Omega_{M P}$ on which jump conditions apply.

A uniform mean flow of constant speed of sound $c_{0}$ follows the direction showed by the arrow in

Fig. 4. In the following equations, $r_{1}^{-}$and $r_{1}^{+}$will then denote the upstream and the downstream part of the MP. Although the cylinder is 3D, the third dimension is considered long in regard to the others so that only radial and azimuthal modes will be considered. 

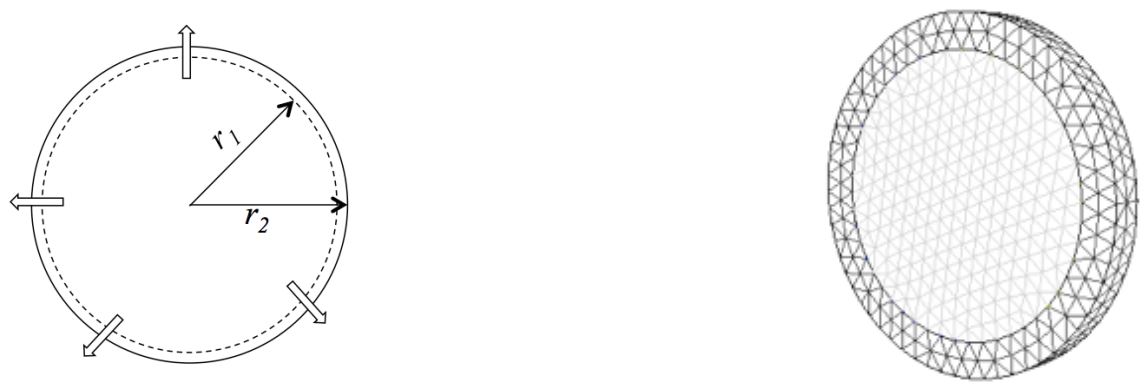

Fig. 4 Academic configuration: cylinder of radius $r_{2}$, with a perforated plate at $r_{1}$, view of the mesh.

For this simple case, Eq. (2) takes the following form,

$$
\left\{\begin{array}{c}
\Delta \hat{p}+k^{2} \hat{p}=0 \text { on } \Omega . \\
\nabla \hat{p} . \mathbf{n}=0 \text { on } \delta \Omega_{W} .
\end{array}\right.
$$

Jump conditions that apply accross $\delta_{M P}$ will be detailed further in Eq. (32) and Eq. (33).

Considering $k^{2}=k_{r}^{2}+k_{z}^{2}\left(k=\frac{\omega}{c_{0}}\right)$, Eq. (24) can be cast in the polar coordinates $(r, \theta, z)$ :

$$
\frac{1}{R} \frac{d^{2}}{d r^{2}} R+\frac{1}{r R} \frac{d}{d r} R+\frac{1}{r^{2} \Theta} \frac{d^{2}}{d^{2} \theta} \Theta+\frac{1}{Z} \frac{d^{2}}{d z} Z+\left(k_{r}^{2}+k_{z}^{2}\right)=0
$$

where $\hat{p}=R(r) \Theta(\theta) Z(z)[2,41]$.

Since we only consider the radial and azimuthal modes, the term in $\frac{d^{2}}{d z^{2}}$ can be neglected so that:

$$
\frac{1}{R} \frac{d^{2}}{d r^{2}} R+\frac{1}{r R} \frac{d}{d r} R+\frac{1}{r^{2}}\left(\frac{1}{\Theta} \frac{d^{2}}{d^{2} \theta} \Theta+n_{\theta}^{2}\right)+k_{r}^{2}-\frac{n_{\theta}^{2}}{r^{2}}=0
$$

Under these conditions, the radial part of Eq. (25) can be reduced to a Bessel equation:

$$
\left(\frac{d^{2}}{d r^{2}} R+\frac{1}{r} \frac{d}{d r} R\right)+R \times\left(k_{r}^{2}-\frac{n_{\theta}^{2}}{r^{2}}\right)=0,
$$

whose general solution is of the form:

$$
R(r)=A J_{n_{\theta}}\left(k_{r} r\right)+B N_{n_{\theta}}\left(k_{r} r\right)
$$

where $J_{n_{\theta}}$ and $N_{n_{\theta}}$ are Bessel functions of order $n_{\theta}$. In the domain $r \leq r_{1}^{-}, N_{n_{\theta}}$ being singular at $r=0$ is put aside and the pressure can then be written:

$$
R(r)=A J_{n_{\theta}}\left(k_{r} r\right) \text { for } r \leq r_{1}^{-} .
$$


In the domain $r_{1}^{+} \leq r \leq r_{2}$, solutions may be written:

$$
R(r)=C J_{n_{\theta}}\left(k_{r} r\right)+D N_{n_{\theta}}\left(k_{r} r\right) .
$$

A wall condition is imposed on the outer cylinder, thus the normal acoustic speed is null on $\delta \Omega$.

The condition $\hat{\mathbf{u}} . \mathbf{n}=0$ at $r=r_{2}$ then leads to:

$$
C J_{n_{\theta}}^{\prime}\left(k_{r} r_{2}\right)+D N_{n_{\theta}}^{\prime}\left(k_{r} r_{2}\right)=0 .
$$

According to Eq. (7), jump conditions can also be written across $\delta_{\Omega_{M P}}$ :

$$
\begin{gathered}
\hat{p}\left(r=r_{1}^{+}\right)-\hat{p}\left(r=r_{1}^{-}\right)=\frac{i \omega \rho d^{2}}{K_{a}} \hat{u}\left(r=r_{1}^{-}\right), \\
\hat{p}\left(r=r_{1}^{-}\right)-\hat{p}\left(r=r_{1}^{+}\right)=-\frac{i \omega \rho d^{2}}{K_{a}} \hat{u}\left(r=r_{1}^{+}\right),
\end{gathered}
$$

where $\hat{u}$ denotes the radial acoustic velocity. We then obtain the system

$$
\mathbf{M X}=0,
$$

where $\mathbf{M}$ is the matrix obtained by using Eq. (31), Eq. (32) and Eq. (33) and is given by

$$
\left[\begin{array}{ccc}
0 & J_{n_{\theta}}^{\prime}\left(k_{r} r_{2}\right) & N_{n_{\theta}}^{\prime}\left(k_{r} r_{2}\right) \\
\frac{d^{2}}{K_{a}} k_{r} J_{n_{\theta}}^{\prime}\left(k_{r} r_{1}^{-}\right)+J_{n_{\theta}}\left(k_{r} r_{1}^{-}\right) & -J_{n_{\theta}}\left(k_{r} r_{1}^{+}\right) & -N_{n_{\theta}}\left(k_{r} r_{1}^{+}\right) \\
J_{n_{\theta}}\left(k_{r} r_{1}^{-}\right) & -J_{n_{\theta}}\left(k_{r} r_{1}^{+}\right)+\frac{d^{2} k_{r}}{K_{a}} J_{n_{\theta}}^{\prime}\left(k_{r} r_{1}^{+}\right) & -N_{n_{\theta}}\left(k_{r} r_{1}^{+}\right)+\frac{d^{2} k_{r}}{K_{a}} N_{n_{\theta}}^{\prime}\left(k_{r} r_{2}\right)
\end{array}\right],
$$

and $X^{t}=\left[\begin{array}{lll}A & C & D\end{array}\right]$.

Solving

$$
\operatorname{det}(\mathbf{M})=0
$$

releases the eigenvalues of the configuration.

\section{B. Results}

Let us consider the following parameters for the multiperforated plate: $U=5 \mathrm{~m} \cdot \mathrm{s}^{-1}, a=3 \mathrm{~mm}$ and $d=35 \mathrm{~mm}, r_{1}=0.2 \mathrm{~m}$ and $r_{2}=0.25 \mathrm{~m}$. The configuration calculated by the Helmholtz solver [36] contains a tetrahedric mesh of 1186 nodes shown in Fig. 4. The sound speed is uniform and equal to $347 \mathrm{~m} \cdot \mathrm{s}^{-1}$. 
The computed eigenfrequencies gathered in Table 1 are compared to the analytical results obtained by solving Eq. (34). The first three modes are considered; they correspond to the first radial $\left(n_{\theta}=0\right)$ and the two first azimuthal $\left(n_{\theta}=1, n_{\theta}=2\right)$ modes.

The Helmholtz solver provides a complex frequency $f=\operatorname{Re}(f)+i \operatorname{Im}(f)$ where $\operatorname{Re}(f)$ is the frequency of the mode, and $\operatorname{Im}(f)$ is related to the amplification rate of the mode. With the adopted convention $p_{1}(\mathbf{x}, t)=\operatorname{Re}\left(\hat{p}(\mathbf{x}) e^{-i \omega t}\right)$, the ratio between the value of the pressure fluctuation at $t=0$ and $T=1 / \operatorname{Re}(f)$ is given by Eq. (35).

$$
\frac{\left|p_{1}(\mathbf{x}, t=T)\right|}{\left|p_{1}(\mathbf{x}, t=0)\right|}=e^{\operatorname{Im}(\omega) T}
$$

It is then useful to define an attenuation factor $\mathcal{A}$ (in percent) over a period $\mathrm{T}$ as $\mathcal{A}=100\left[1-e^{2 \pi \frac{\operatorname{Im}(f)}{R e(f)}}\right]$.

\begin{tabular}{|c|c|c|c|c|}
\hline & \multicolumn{2}{|c|}{ Numerical results } & \multicolumn{2}{c|}{ Analytical results } \\
\hline \hline & $\operatorname{Re}(f)$ & $\operatorname{Im}(f)(\mathcal{A} \%)$ & $\operatorname{Re}(f)$ & $\operatorname{Im}(f)(\mathcal{A} \%)$ \\
\hline$n_{\theta}=1$ & $382.5 \mathrm{~Hz}$ & $\mathbf{- 1 8 . 8} s^{-1}(26.7 \%)$ & $382.6 \mathrm{~Hz}$ & $\mathbf{- 1 8 . 9} s^{-1}(27.8 \%)$ \\
$n_{\theta}=0$ & $534.1 \mathrm{~Hz}$ & $\mathbf{- 9 7 . 5} s^{-1}(68.2 \%)$ & $533.2 \mathrm{~Hz}$ & $\mathbf{- 9 7 . 5} s^{-1}(68.3 \%)$ \\
$n_{\theta}=2$ & $610.5 \mathrm{~Hz}$ & $\mathbf{- 2 1 . 4} s^{-1}(19.8 \%)$ & $611 \mathrm{~Hz}$ & $\mathbf{- 2 1 . 6} s^{-1}(19.9 \%)$ \\
\hline
\end{tabular}

Table 1 Comparison of the eigenfrequencies between numerical and analytical results.

As expected, the eigenfrequencies have a negative imaginary part, which means that the pressure fluctuation is damped. The numerical results are in good agreement with the theory.

\section{Results on the first azimuthal mode}

Figure 5 shows the radial profiles of the real and imaginary parts of $\hat{p}$ given by the Helmholtz solver for the first azimuthal mode $\left(n_{\theta}=1\right)$ plotted against analytical results for comparison. Again, a good agreement is found. The 2D pressure distribution released by the Helmholtz solver as well as isolines of pressure is also given. Note that the pressure jump across the perforated plate is visible 
on the real and imaginary parts of the harmonic pressure fluctuations, as well as on the discontinuity of the isolines.

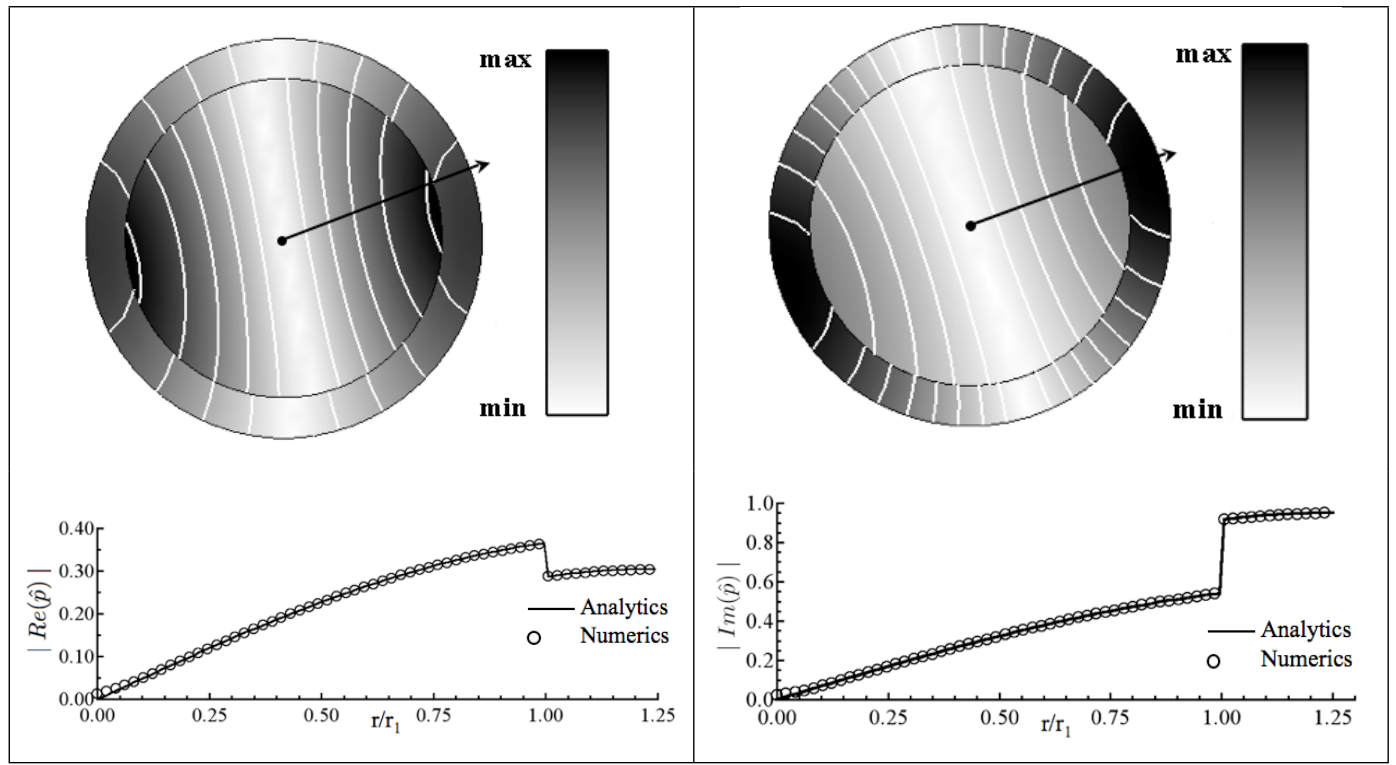

Fig. 5 First azimuthal mode: $n_{\theta}=1, \operatorname{Re}(f)=382.5 \mathrm{~Hz}, \operatorname{Im}(f)=\mathbf{- 1 8 . 8} \mathrm{s}^{-1}$. Left: Re( $\left.\hat{p}\right)$. Right: Im $(\hat{p})$. Above : Numerical pressure distribution and isolines. Below: Radial profiles of the real and imaginary part of $\hat{p}$ along the radius represented by the arrow (comparison Analytics/Numerics).

This comparison was conducted for a fixed bias flow speed $\left(5 \mathrm{~m} . \mathrm{s}^{-1}\right)$, but Figure 6 also shows good agreement between numerical and analytical results for various bias flow speeds.

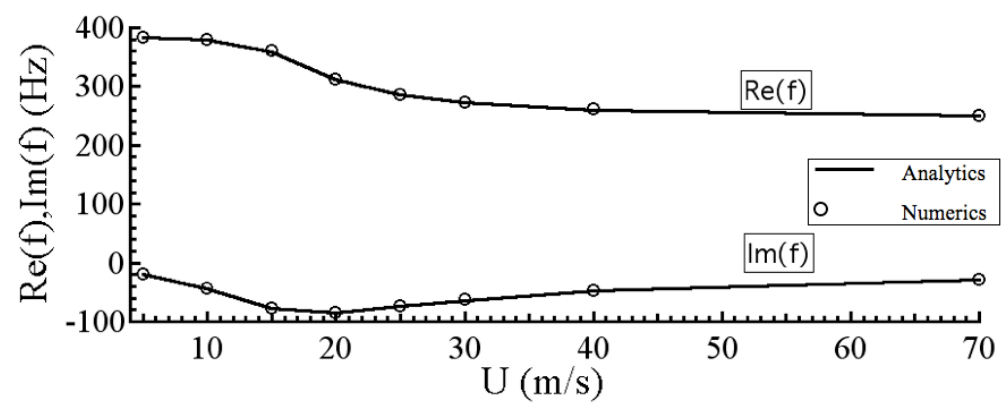

Fig. 6 Comparison between numerical and analytical results. $\operatorname{Re}(f)$ and $\operatorname{Im}(f)$ are shown for various speeds for the first azimuthal mode. 


\section{Results on the radial mode}

Figure 7 shows the fields of pressure of the radial mode $\left(n_{\theta}=0\right)$. A good agreement between numerical and analytical results is found. Unlike the first azimuthal mode, no discontinuity is observed on the isolines of pressure, due to the form of the pressure field.

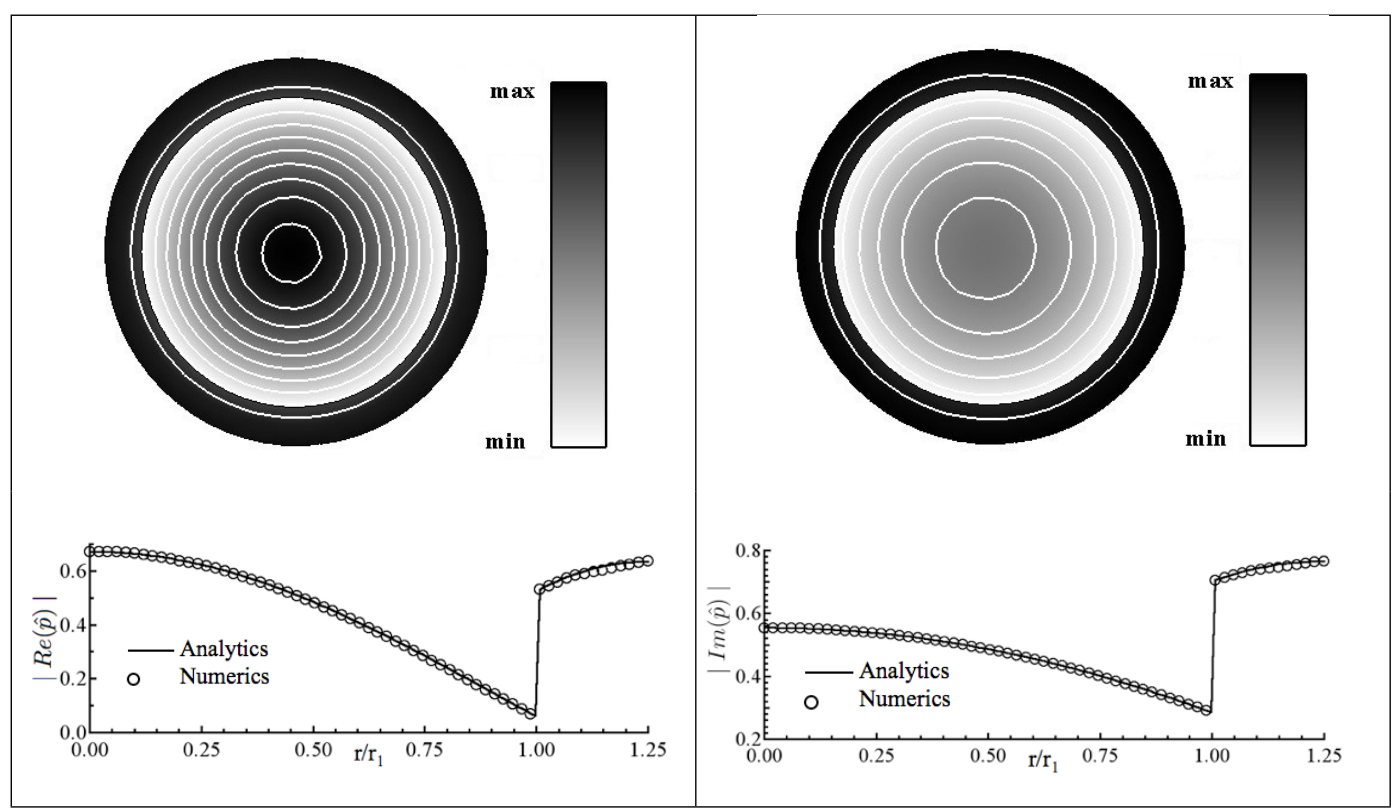

Fig. 7 Radial mode: $n_{\theta}=0, \operatorname{Re}(f)=534.1 \mathrm{~Hz}, \operatorname{Im}(f)=\mathbf{9 7 . 5} \mathrm{s}^{-1}$. Left: $\operatorname{Re}(\hat{p})$. Right: $\operatorname{Im}(\hat{p})$. Above : Numerical pressure distribution and isolines. Below: Radial profiles of the real and imaginary part of $\hat{p}$ (comparison Analytics/Numerics).

\section{Comparison of the evolution of both modes against bias flow speed}

Figure 8 shows the evolution of the resonant frequencies for the radial mode and the first azimuthal mode as a function of the bias flow speed. As the bias flow speed increases, the resonant frequency of both modes decreases. But in the case of the azimuthal mode, the resonant frequencies varies in a range from $380 \mathrm{~Hz}$ to $260 \mathrm{~Hz}$, whereas the frequency of the radial mode varies a lot, droping from $500 \mathrm{~Hz}$ to $80 \mathrm{~Hz}$.

The frequency of the radial mode hence becomes close to the one of the azimuthal mode and then is lower than it. Figure 9 displays the attenuation factor of the radial and the first azimuthal mode as a function of the bias flow speed. In the case of the radial mode, the damping raises until reaching almost $100 \%$ and keeping this very high value of damping. 


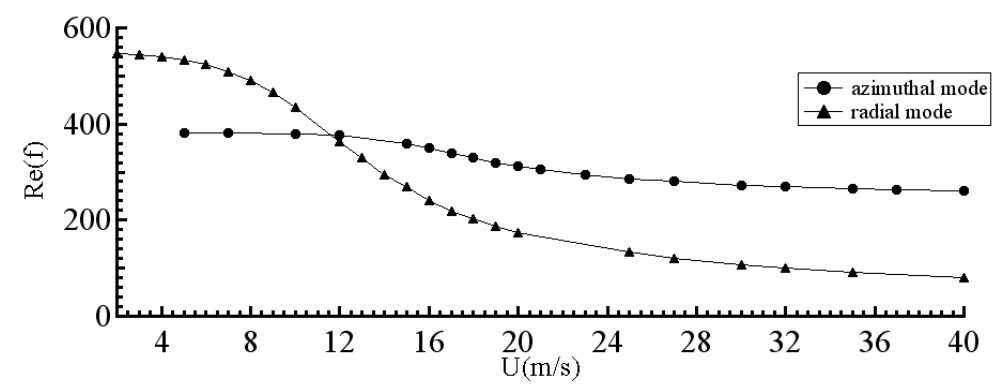

Fig. 8 Resonant frequencies as a function of the bias flow speed for the radial and the first azimuthal mode. Symbols are for numerical results while the line stands for analytical results.

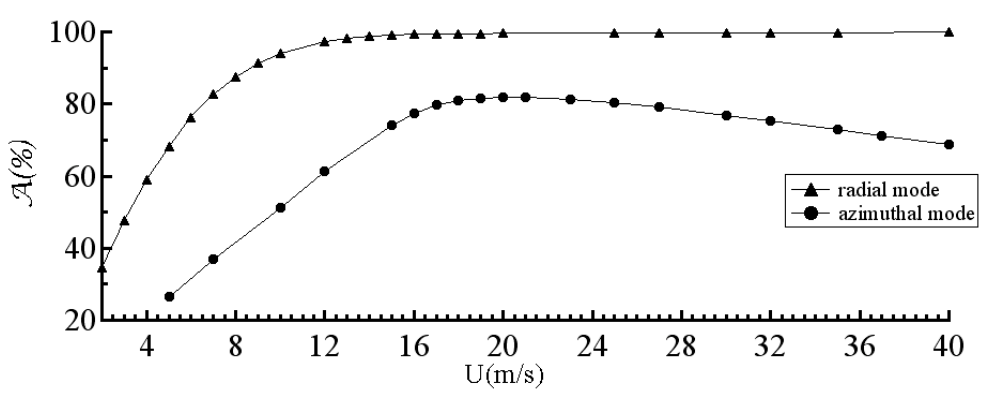

Fig. 9 Attenuation factor as a function of the bias flow speed $U$ for the radial and the first azimuthal mode. Symbols are for numerical results while the line stands for analytical results.

On the contrary, the damping for the first azimuthal mode reaches a maximum at $U_{m d} \approx 20 \mathrm{~m} \cdot \mathrm{s}^{-1}$ and then decreases. For this particular simple geometry, the impact of the perforated plates is thus more important on radial modes than on azimuthal modes. For the radial mode, the pressure jump is constant over all the perimeter, which makes the pressure drop important everywhere, and thus, according to Eq. (14), the perforated plate has a strong impact. This will be further explained in Section IV B with an energy analysis.

\section{Energy analysis}

\section{A. General analysis}

In order to evaluate the dissipation induced by perforated plates, an analysis of the energy budget is done for the cylinder configuration. For a non reactive case, the conservation equation for 
the acoustic energy $E(\mathbf{x}, t)=\frac{1}{2} \rho \mathbf{u}_{\mathbf{1}}^{2}(\mathbf{x}, t)+\frac{1}{2 \gamma p_{0}} p_{1}^{2}(\mathbf{x}, t)$ can be written as follows:

$$
\frac{\partial E}{\partial t}+\nabla \cdot p_{1} \mathbf{u}_{1}=0
$$

Integrating Eq. (36) over the flow domain and a time period T yields to:

$$
\frac{1}{T} \int_{T} \frac{\partial \bar{E}}{\partial t} \mathrm{dt}=-\frac{1}{\mathrm{~T}} \int_{\mathrm{T}} \mathcal{F}_{1}
$$

where $\bar{E}=\iiint_{\Omega} E d \Omega$ is the volume integrated acoustic energy and $\delta \Omega$ can be split into four parts: $\delta \Omega_{Z}$ where a finite valued impedance is imposed, $\delta \Omega_{M P}$ where a MP condition is imposed, $\delta \Omega_{W}$ where an impermeable wall condition is prescribed and $\delta \Omega_{P}$ where the acoustic pressure is set to zero. The term $\mathcal{F}_{1}$ is the sum of the acoustic fluxes over the boundaries, defined by:

$$
\mathcal{F}_{1}=\iint_{\delta \Omega} p_{1} \mathbf{u}_{\mathbf{1}} \cdot \mathbf{n} \mathrm{d} S
$$

Combining the momentum equation $\rho i \omega \hat{\mathbf{u}} \cdot \mathbf{n}=\boldsymbol{\nabla} \hat{p} . \mathbf{n}$ and Eq. (22), the following relation between the acoustic velocity fluctuation and the acoustic pressure jump over a MP is derived:

$$
\hat{\mathbf{u}} \cdot \mathbf{n}=\frac{-1}{\rho^{-} i \omega} \frac{K_{a}}{d^{2}}[\hat{p}]
$$

As for $\delta \Omega_{Z}$, combining the momentum equation with Eq. (3), we obtain

$$
\hat{\mathbf{u}} \cdot \mathbf{n}=\frac{\hat{p}}{\rho c Z}
$$

Using Eq. (40) and Eq. (39) to express the flux, the acoustic energy budget reads:

$$
\frac{\partial \bar{E}}{\partial t}=-\iint_{\delta \Omega_{Z}} \operatorname{Re}\left(\hat{p}(\mathbf{x}) e^{-i \omega t}\right) \operatorname{Re}\left(\frac{\hat{p}(\mathbf{x})}{\rho c Z} e^{-i \omega t}\right) \mathrm{d} S-\iint_{\delta \Omega_{M P}} \operatorname{Re}\left(\hat{p}(\mathbf{x}) e^{-i \omega t}\right) \operatorname{Re}\left(\frac{-1}{\rho^{-} i \omega} \frac{K_{a}}{d^{2}}[\hat{p}] e^{-i \omega t}\right) \mathrm{d} S
$$

The contribution of the flux over $\delta \Omega_{W}$ and $\delta \Omega_{P}$ is null because either $p_{1}$ or $\mathbf{u}_{\mathbf{1}} \cdot \mathbf{n}$ vanishes. Since there is no impedance condition, the energy loss only occurs through the perforated plates. After integrating over one period $\mathrm{T}$, we have:

$$
\frac{\bar{E}(t=T)}{\bar{E}(t=0)}-1=-\frac{1}{\bar{E}(t=0)} \int_{T} \iint_{\delta \Omega_{M P}} \operatorname{Re}\left(\hat{p}(\mathbf{x}) e^{-i \omega t}\right) \operatorname{Re}\left(\frac{-K_{a}}{d^{2} \rho^{-} i \omega}[\hat{p}] e^{-i \omega t}\right) \mathrm{d} S
$$


and after some algebra,

$\int_{0}^{T} p_{1} \mathbf{u}_{\mathbf{1}} \cdot \mathbf{n} \mathrm{d} t=$

$$
\begin{aligned}
& \frac{1}{\rho\left(\omega_{r}^{2}+\omega_{i}^{2}\right)}\left[\omega_{i} \operatorname{Im}\left(\frac{K_{a}}{d^{2}}\right)+\omega_{r} \operatorname{Re}\left(\frac{K_{a}}{d^{2}}\right)\right] \\
& {\left[-A \operatorname{Re}(\hat{p}) \operatorname{Im}\left(p^{+}-p^{-}\right)+B \operatorname{Re}\left(p^{+}-p^{-}\right) \operatorname{Im}(\hat{p})+C\left(\operatorname{Re}(\hat{p}) \operatorname{Re}\left(p^{+}-p^{-}\right)-\operatorname{Im}\left(p^{+}-p^{-}\right) \operatorname{Im}(\hat{p})\right)\right]} \\
& +\frac{1}{\rho\left(\omega_{r}^{2}+\omega_{i}^{2}\right)}\left[\omega_{i} \operatorname{Re}\left(\frac{K_{a}}{d^{2}}\right)-\omega_{r} \operatorname{Im}\left(\frac{K_{a}}{d^{2}}\right)\right] \\
& {\left[A \operatorname{Re}(\hat{p}) \operatorname{Im}\left(p^{+}-p^{-}\right)+B \operatorname{Im}(\hat{p}) \operatorname{Im}\left(p^{+}-p^{-}\right)+C\left(\operatorname{Im}(\hat{p}) \operatorname{Re}\left(p^{+}-p^{-}\right)+\operatorname{Re}(\hat{p}) \operatorname{Im}\left(p^{+}-p^{-}\right)\right)\right],}
\end{aligned}
$$

with $\mathrm{A}, \mathrm{B}$, and $\mathrm{C}$ defined as follows:

$$
\left\{\begin{array}{ccc}
C=\int_{0}^{T} e^{2 \omega_{i} t} \cos \left(\omega_{r} t\right) \sin \left(\omega_{r} t\right) d t=\frac{\omega_{r}\left(1-e^{2 \omega_{i} T}\right)}{4\left(\omega_{r}^{2}+\omega_{i}^{2}\right)} & \text { and if } \omega_{i}=0, \quad C=0, \\
A=\int_{0}^{T} e^{2 \omega_{i} t} \cos ^{2}\left(\omega_{r} t\right) d t=\frac{e^{2 \omega_{i} T}-1}{2 \omega_{i}}+\frac{\omega_{r}}{\omega_{i}} C \quad \text { and if } \omega_{i}=0, \quad A=\frac{T}{2}, \\
B=\int_{0}^{T} e^{2 \omega_{i} t} \sin ^{2}\left(\omega_{r} t\right) d t=-\frac{\omega_{r}}{\omega_{i}} C=\frac{\omega_{r}^{2}\left(e^{2 \omega_{i} T}-1\right)}{4 \omega_{i}\left(\omega_{r}^{2}+\omega_{i}^{2}\right)} & \text { et si } \omega_{i}=0, \quad B=\frac{T}{2} .
\end{array}\right.
$$

The contribution of a MP can be obtained by summing the fluxes on both sides of the plate. An analytical expression can be derived in the simple case where $\omega_{i} \ll \omega_{r}$ and $\hat{u}$ do not vary along the tangential direction to the MP. Considering a perforated plate composed of two boundaries as shown in Fig. 10, an asymptotic formulation can be derived for the term $\mathcal{F}_{1}$ of Eq. (38) following the conventions defined in Fig. 10 where $\delta V$ is the boundary of a control volume of small size compared to the acoustic wavelength $\lambda$.

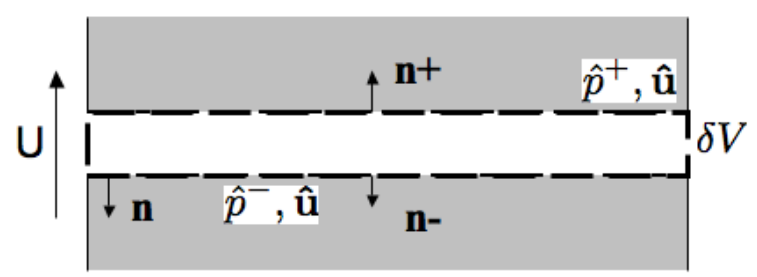

Fig. 10 Calculation of the flux difference across a perforated plate.

Its expression is:

$$
\mathcal{F}_{1}=\oint_{\delta \Omega} \operatorname{Re}\left(\hat{p}^{+} e^{-i \omega t}\right) \operatorname{Re}\left(\hat{\mathbf{u}}^{+} \cdot \mathbf{n}^{+} e^{-i \omega t}\right)+\operatorname{Re}\left(\hat{p}^{-} e^{-i \omega t}\right) \operatorname{Re}\left(\hat{\mathbf{u}}^{-} \cdot \mathbf{n}^{-} e^{-i \omega t}\right) \mathrm{d} S .
$$

Then making use of the fact that $\Delta \ll \lambda$, the flux density is simply

$$
F_{1}=\frac{\mathcal{F}_{1}}{\Delta}=\operatorname{Re}\left(\hat{p}^{+} e^{-i \omega t}\right) \operatorname{Re}\left(\hat{\mathbf{u}}^{+} \cdot \mathbf{n}^{+} e^{-i \omega t}\right)+\operatorname{Re}\left(\hat{p}^{-} e^{-i \omega t}\right) \operatorname{Re}\left(\hat{\mathbf{u}}^{-} \cdot \mathbf{n}^{-} e^{-i \omega t}\right) .
$$


Integrating over a period $T$ leads to:

$$
\frac{1}{T} \int_{T} F_{1} \mathrm{~d} t=\frac{1}{T} \int_{T} \operatorname{Re}\left(\hat{p}^{+} e^{-i \omega t}\right) \operatorname{Re}\left(\hat{\mathbf{u}}^{+} \cdot \mathbf{n}^{+} e^{-i \omega t}\right)+\operatorname{Re}\left(\hat{p}^{-} e^{-i \omega t}\right) \operatorname{Re}\left(\hat{\mathbf{u}}^{-} \cdot \mathbf{n}^{-} e^{-i \omega t}\right) \mathrm{d} t .
$$

Since $\hat{u}^{+}=\hat{u}^{-}=\hat{u}$ and $\mathbf{n}^{+}$and $\mathbf{n}^{-}$point to opposite directions, using Eq. (39) then leads to:

$$
\frac{1}{T} \int_{T} F_{1} \mathrm{~d} t=\int_{T} \operatorname{Re}\left(\left[\hat{p}^{+}-\hat{p}^{-}\right] e^{-i \omega t}\right) \operatorname{Re}\left(\frac{K_{a}}{\rho^{-} i \omega d^{2}}\left[\hat{p}^{+}-\hat{p}^{-}\right] e^{-i \omega t}\right) \mathrm{d} t
$$

The hypothesis $\omega_{i} \ll \omega_{r}$ yields to

$$
\frac{1}{T} \int_{T} F_{1} \mathrm{~d} t=\frac{1}{2} \operatorname{Re}\left(\left[\hat{p}^{+}-\hat{p}^{-}\right]^{*} \frac{K_{a}}{\rho^{-} i \omega d^{2}}\left[\hat{p}^{+}-\hat{p}^{-}\right]\right)
$$

where ()$^{*}$ denotes the conjugate of a complex number. Then, it yields:

$$
\frac{1}{T} \int_{T} F_{1} \mathrm{~d} t=\frac{-\left|\hat{p}^{+}-\hat{p}^{-}\right|^{2}}{2 \rho^{-} \omega_{r} d^{2}} \operatorname{Re}\left(i K_{a}\right)
$$

Using Eq. (8)

$$
\frac{1}{T} \int_{T} F_{1} \mathrm{~d} t=-\frac{\left|\hat{p}^{+}-\hat{p}^{-}\right|^{2}}{\rho^{-} \omega_{r} d^{2}} a \Delta_{a}
$$

which is negative for all frequencies as soon as $\Delta_{a}>0$. The net flux $\mathcal{F}_{1}$ towards the control volume Fig. 10 is then positive (recall that the $\mathbf{n}$ vector points outwards the control volume of Fig. 10), inducing a loss of energy for the fluid domain surrounding the MP. Thus any acoustic model with positive $\Delta_{a}$ generates some amount of damping for the acoustic modes. This result completes the result showed by Howe [42] in the case of a uniform density.

\section{B. Numerical calculation of dissipation through the perforated plates of the cylinder.}

The left and right hand side of Eq. (41) were assessed numerically for the radial and the azimuthal modes for two values of the bias flow speed: $U=5 \mathrm{~m} \cdot \mathrm{s}^{-1}$ and $U=20 \mathrm{~m} \cdot \mathrm{s}^{-1}$. Note that the error of $0.06 \%$ caused by the Mach value of the bias flow speed of $U=20 \mathrm{~m}_{\mathrm{s}} \mathrm{s}^{-1}$ is considered negligeable in front of the approximations made in this thermoacoustic study. The results are gathered 
in Table 2. A new damping factor $\mathrm{A}_{e n}$ is now defined as $\left|\frac{\bar{E}(t=T)}{E(t=0)}-1\right|$. This damping factor refers to the damping of the acoustic energy whereas the damping factor defined previously in section III B refered to the damping of the pressure amplitude only. In the case of the first azimuthal mode $\left(U=5 \mathrm{~m} . \mathrm{s}^{-1}\right)$, the term $\mathrm{A}_{e n}$ is found to be $46.19 \%$ while $-\int_{T} \iint_{\delta \Omega_{M P}} p_{1} \mathbf{u}_{\mathbf{1}} \cdot \mathbf{n} \mathrm{dS}$ gives $-46.37 \%$. In the table, the normalized error defined by

$$
\epsilon=\frac{\left|\frac{\bar{E}(t=T)}{\bar{E}(t=0)}-1+\int_{T} \iint_{\delta \Omega_{M P}} p_{1} \mathbf{u}_{\mathbf{1}} \cdot \mathbf{n} d S\right|}{|\bar{E}(t=0)|}
$$

is also given; the equation of acoustic energy is well balanced for the two modes for $U=5 m . s^{-1}$.

\begin{tabular}{|c|c|c|c|}
\hline & $\frac{\bar{E}(t=T)}{\bar{E}(t=0)}-1$ & $-\int_{T} \iint_{\delta \Omega_{M P}} p_{1} \mathbf{u}_{\mathbf{1}} \cdot \mathbf{n} \mathrm{dS}$ & $\epsilon$ \\
\hline First azimuthal mode $U=5 \mathrm{~m} . \mathrm{s}^{-1}$ & $-46.19 \%$ & $-46.37 \%$ & $0.4 \%$ \\
\hline First azimuthal mode $U=20 \mathrm{~m} \cdot \mathrm{s}^{-1}$ & $-96.73 \%$ & $-96.82 \%$ & $0.096 \%$ \\
\hline Radial mode $U=5 \mathrm{~m} . \mathrm{s}^{-1}$ & $-89.8 \%$ & $-92 \%$ & $2.5 \%$ \\
\hline Radial mode $U=20 \mathrm{~m} \cdot \mathrm{s}^{-1}$ & $-99.65 \%$ & $-99.69 \%$ & $0.036 \%$ \\
\hline
\end{tabular}

Table 2 Acoustic energy analysis on the cylinder.

The same is true when considering the bias flow velocity corresponding to the maximal damping as found for the modes in Section III B. In both cases, the damping increases: with a bias flow speed of $U_{m d}=20 \mathrm{m.s} \mathrm{s}^{-1}$, the azimuthal mode is damped by $96.7 \%$ while $A_{e n}=99.65 \%$ is reached for the radial mode.

Figure 11 shows $\left|\hat{p}^{+}-\hat{p}^{-}\right|$on each point of the MP, adimensionalized by the maximum of fluctuating pressure over the domain against the angle $\theta$ for the azimuthal mode at $U=5 m . s^{-1}$ and $U=U_{m d}$. The same value is also plotted at $U_{m d}$ for the radial mode. The profile of $\left|\hat{p}^{+}-\hat{p}^{-}\right|$of the radial mode is constant over all the MP, whereas for the azimuthal mode, $\left|\hat{p}^{+}-\hat{p}^{-}\right|$varies along the curvilinear abscissa and is globaly smaller than for the radial fluctuation. According to Eq. (50), the higher $\left|\hat{p}^{+}-\hat{p}^{-}\right|$is, the higher the dissipation is; thus Fig. 11 is fully consistent with the results of Tab. 2. Figure 11 also shows that the pressure jump for the azimuthal mode at $U=20 \mathrm{~m}^{-s^{-1}}$ is globally higher than for the case at $U=5 \mathrm{~m} . \mathrm{s}^{-1}$, which explains the increase in the damping when $\mathrm{U}$ is equal to $U_{m d}$. 


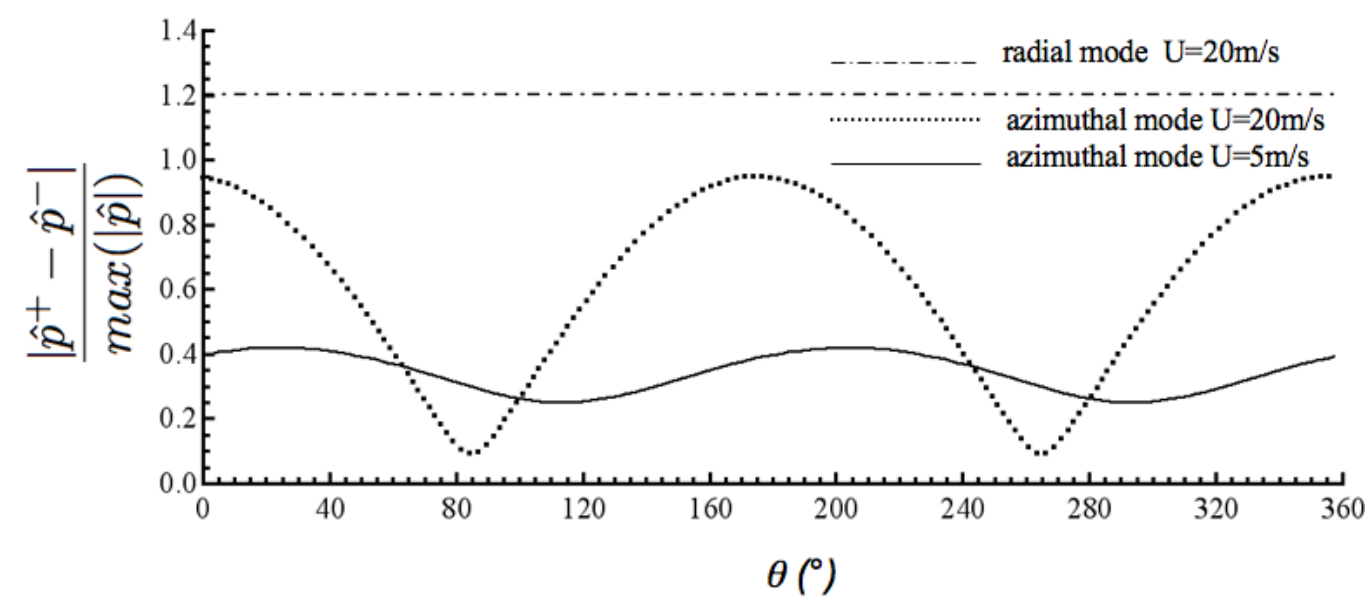

Fig. $11 \frac{\left|p^{+}-p^{-}\right|}{\max (\hat{p})}$ as a function of $\theta$ for the radial and the first azimuthal mode.

\section{Acoustic energy analysis of an industrial helicopter chamber}

The purpose of this section is to illustrate the flexibilily of the methodology described in the paper by computing a 3D actual industrial chamber. Unlike the previous academic test case, the chamber contains several MPs. An analysis of the energy budget is therefore carried out in order to evaluate which perforated plates are the most responsible for the damping of the mode as well as to assess the error made on the computations of the acoustic modes. Note that some details regarding the MP geometry and bias flow are omitted for confidentiality reasons. Still, enough information is provided to illustrate the applicability of the method. At last, Howe's model was used for the runs presented in the remaining of this paper as typical of the models discussed in Section II C. Note that since no detailed experimental data are available for the industrial case presented in the following, this choice does not result from a model selection based on quantitative comparisons. Using another model would lead to slightly different results but would neither change the trends presented below nor the illustration of the usefulness of such models in Helmholtz solvers. For example, the modified Howe model developped by Jing and Sun [33] differs by $0.8 \%$ on the imaginary part, which doesn't change the behaviour. 


\section{A. Description of the chamber and first results}

The chamber studied here is a reverse-flow annular combustor and is displayed in Fig. 12. The air inlet comes out of the compressor stage through the casing. Some of this air is used to cool the chamber walls by two means: perforated plates and cooling films. Several jets let into the chamber by the orifices of these cooling devices coalesce to form a cooling coat of air on the inside walls which protects them. The whole chamber contains fifteen burners. As a first step, the computational domain is thus limited to a 24-degree sector of the chamber, corresponding to one burner. It includes the swirler, the casing and the burner. Computations of the whole geometry with the fifteen sectors will be discussed in Section VI. Figure 12 shows the location of the cooling devices on a sector of the chamber: the cooling films are denoted by the letter $\mathrm{F}$, and the different perforated plates are denoted by MP1, MP2 and MP3. The index i stands for interior and corresponds to the plates in the inside part of the chamber. As shown in Fig. 12, the surface area of the different MP zones are different. The holes have the same diameter, but the bias flow speed is not the same for all plates and is computed using data on flow rates. The inlet of air and the outlet are considered in this computation as acoustically closed conditions, as well as all the chamber walls.

The mesh used contains approximatively 450000 nodes and 2.500.000 tetrahedric cells. A cut of the mesh is shown in Fig. 14, while Fig. 15 shows the mesh on the perforated plates. The mesh is quite regular, with a ratio of less than 200 between the maximum and the minimum volume of the mesh cells. The sound speed field used by the Helmholtz solver illustrated in Fig. 13; it is deduced from a previous Large-Eddy Simulation [8] which is subsequently interpolated over the mesh used for the acoustic computation.

Note that this chamber has already been computed in previous studies: for example the influence of the geometry on the acoustics of this chamber was previously studied [15] and several computational fluid dynamics calculations were conducted [7, 43]. The influence of the presence of perforated plates has previously been studied by the authors [40]. It was shown that when using the actual parameters of bias flow through the perforated plates, a very slight influence of the plates on the first longitudinal mode is found. 

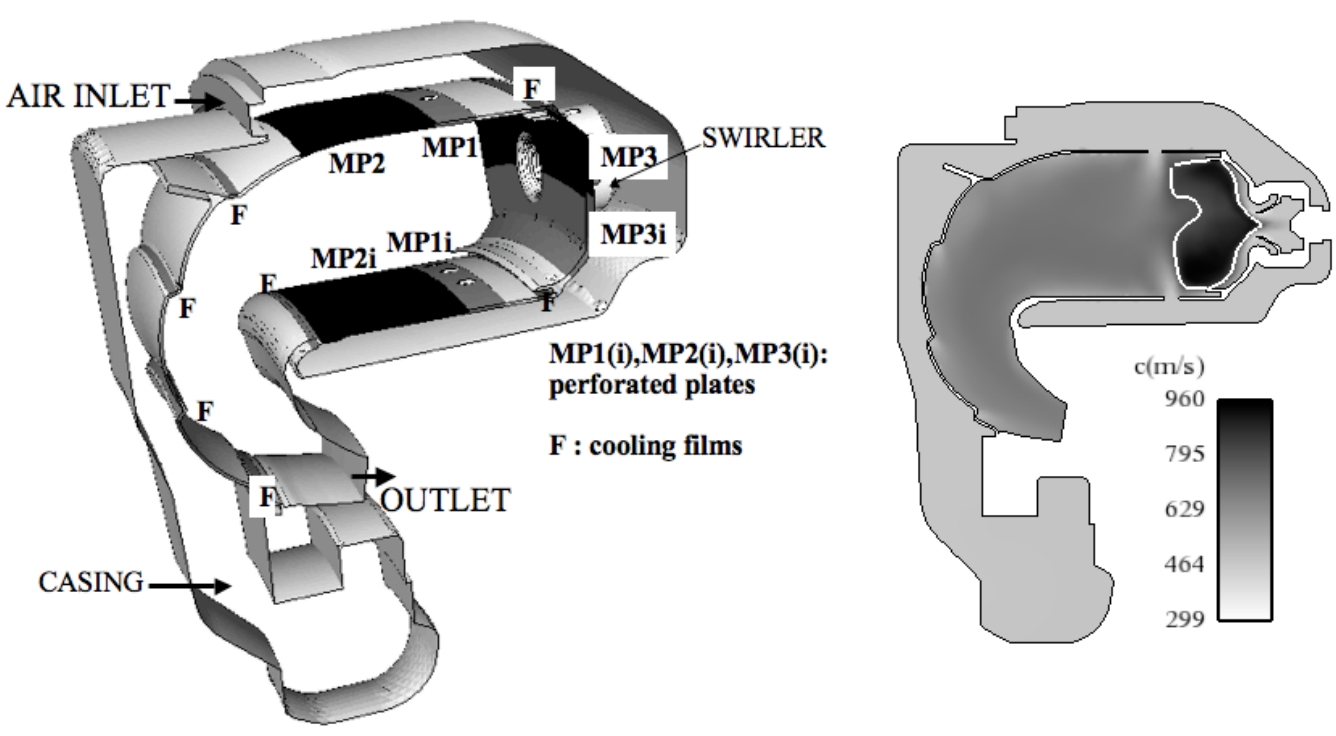

Fig. 13 Sound speed field.

Fig. 12 Location of the MP in the chamber.
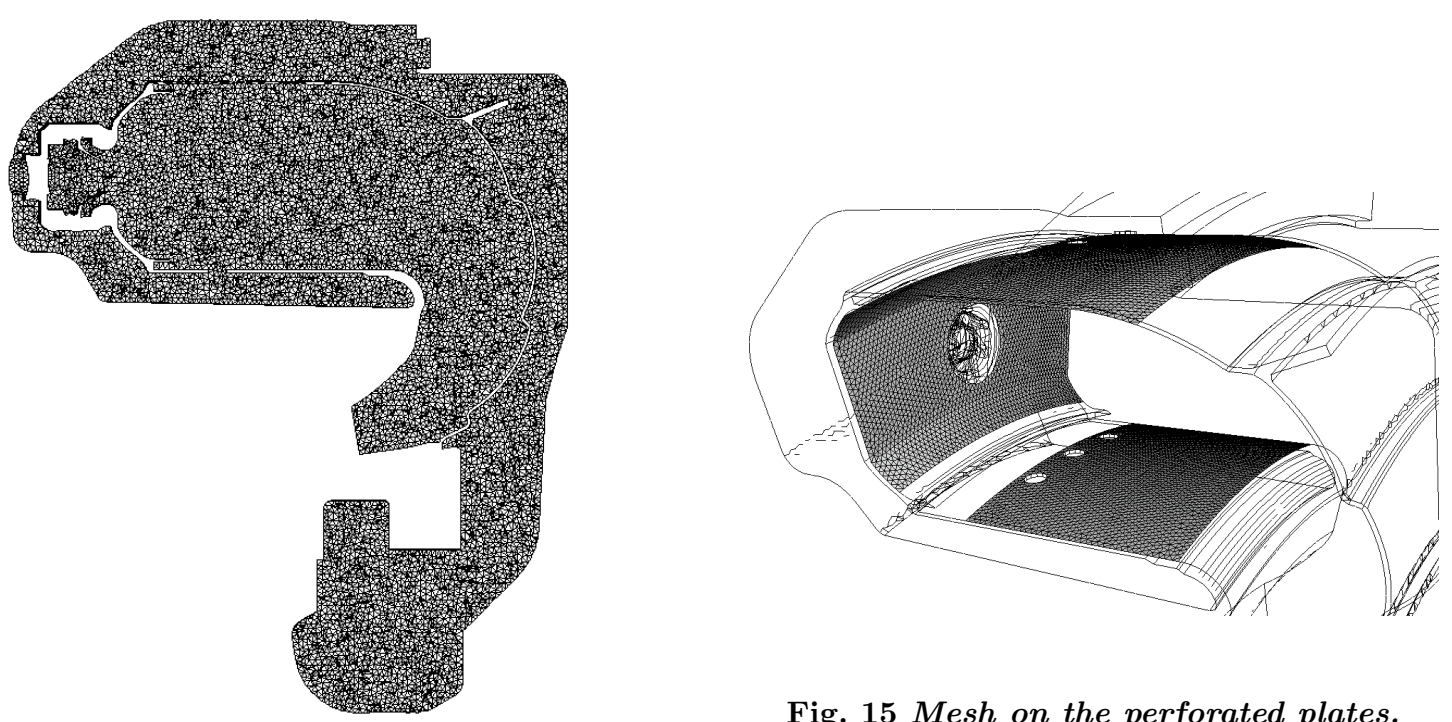

Fig. 15 Mesh on the perforated plates.

Fig. 14 Mesh view on a cut in the middle

of the chamber.

The mode structure is hardly changed and the damping is weak. A typical distribution of the acoustic pressure field is given in Fig. 16. The second longitudinal mode was found more damped, due to the structure of the mode as shown in Table 3 . Indeed the pressure drop between the casing and the combustion chamber is more important for the second mode, and well located to be efficient for the MP. 


\begin{tabular}{|c|c|c|c|c|}
\hline & \multicolumn{2}{|c|}{$1 \mathrm{~L}$} & \multicolumn{2}{c|}{$2 \mathrm{~L}$} \\
\hline \hline & $\operatorname{Re}(f)$ & $\operatorname{Im}(f)$ & $\operatorname{Re}(f)$ & $\operatorname{Im}(f)$ \\
\hline & 507.94 & $-2.65 \mathrm{~Hz}$ & $1150.98 \mathrm{~Hz}$ & $-41.95 \mathrm{~Hz}$ \\
\hline$A_{e n}$ & $6.3 \%$ & \multicolumn{2}{|c|}{$37.3 \%$} \\
\hline
\end{tabular}

Table 3 Frequencies of the first two longitudinal modes (1L and 2L)
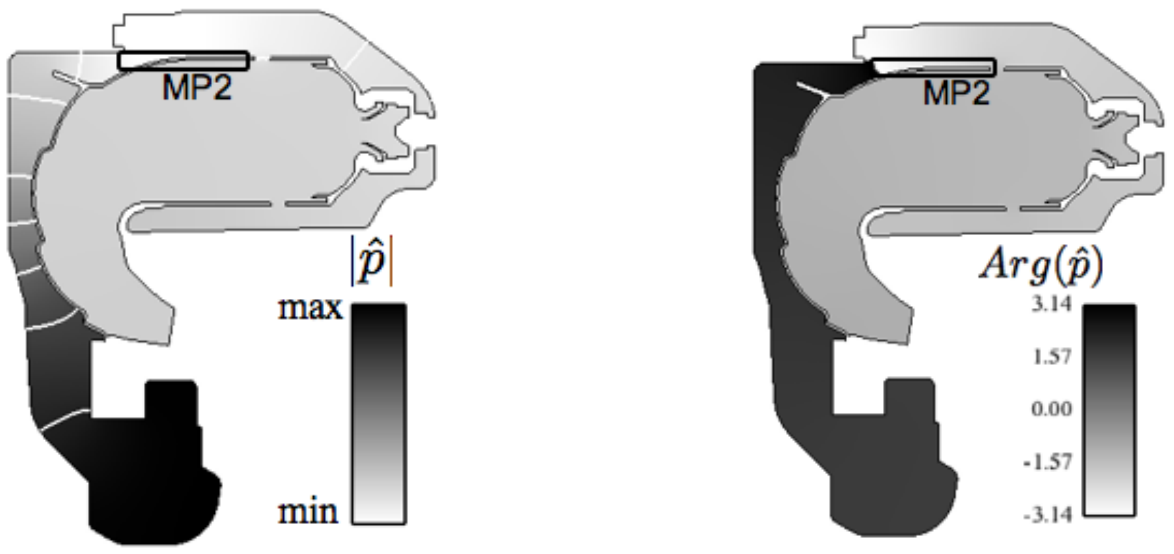

Fig. 16 Field of $|\hat{p}|$ and $\operatorname{Arg}(\hat{p})$ for the $1 \mathrm{~L}$ mode (the square shows the location of the plate MP2).

B. Energy analysis of the first longitudinal mode (1L)

1. Spatial structure can stand for the efficiency of plates

An energy budget is conducted for the first longitudinal mode. The two terms of Eq. (41) can be assessed by post-processing the results from the Helmholtz solver and for the right hand side term, each contribution of each perforated plate is computed. Results are gathered in Table 4 where all contributions are normalized by the energy of the mode integrated over the domain at $t=0$ $\left(\bar{E}_{1}(t=0)\right)$ so that we have:

$$
e^{2 \omega_{i} T}-1+(M P 1+M P 1 i+M P 2+M P 2 i+M P 3+M P 3 i)=0,
$$

where $M P i$ is the value of the contribution of plate $M P k$. 


\begin{tabular}{|c|c|c|c|c|c|c|c|c|}
\hline 1L & $e^{2 \omega_{i} T}-1$ & $\epsilon$ & MP1 & MP1i & MP2 & MP2i & MP3 & MP3i \\
\hline \hline all MPs & -6.4 & 0.36 & 0.625 & $4.5 .10^{-3}$ & $\mathbf{5 . 3 6}$ & $1.7 .10^{-2}$ & 0.526 & 0.186 \\
\hline & & & 9.30 & 0.067 & $\mathbf{7 9 . 7 8}$ & 0.25 & 7.83 & 2.77 \\
\hline no MP2 & -1.1 & 0.2 & 0.637 & $4.41 .10^{-3}$ & - & $1.7 .10^{-2}$ & 0.541 & 0.195 \\
\hline & & & 45.68 & 0.32 & - & 1.22 & 38.8 & 13.98 \\
\hline
\end{tabular}

Table 4 Acoustic energy budget on 1L mode (all values in \%).

$\epsilon$ is the normalized error between the LHS and RHS of Eq. (52). The contribution of each plate to the total damping is also indicated. For example, the plate MP2 contributes to aproximately $80 \%$ of the total damping, while its actual damping $A_{e n}$ is of $5.4 \%$. The damping of the $1 \mathrm{~L}$ mode is of $6.4 \%$ by period T. The energy balance is well closed with a normalized error of $0.36 \%$. Table 4 shows that the plate MP2 is the most efficient at damping. It is necessary to look at the pressure distribution for the $1 \mathrm{~L}$ mode to understand this behavior. The distribution of $|\hat{p}|$ is given in Fig. 16 . The pressure node is roughly located above MP2 (see the phase shift in Fig. 16) inducing the pressure drop to be maximum across plate MP2, thus inducing a significant damping from this very plate (see Eq. (50)).

Since it is responsible for almost the totality of the damping, another case is run without plate MP2 to see if it modifies the contribution of the other plates. A null acoustic speed condition is applied in this case on plate MP2. The frequency of the mode is hardly changed. The damping is now of $1 \%$. This is almost equal to the previous damping minus the contribution of plate MP2. Since the imaginary part of the mode is now small $(\operatorname{Im}(f)=-0.46 \mathrm{~Hz})$, the mode can be said marginally stable. The contribution of the other plates is hardly changed: this can be explained by the fact that at the actual regime, the presence of the MPs doesn't have a strong impact on the mode structure. Figure 17 shows that replacing the plate MP2 by a wall has hardly changed the pressure field of the mode. The pressure node remains above plate MP2, preventing the other plates from improving their damping effect. The damping cannot be assured by the other plates, and the mode becomes marginally stable. 

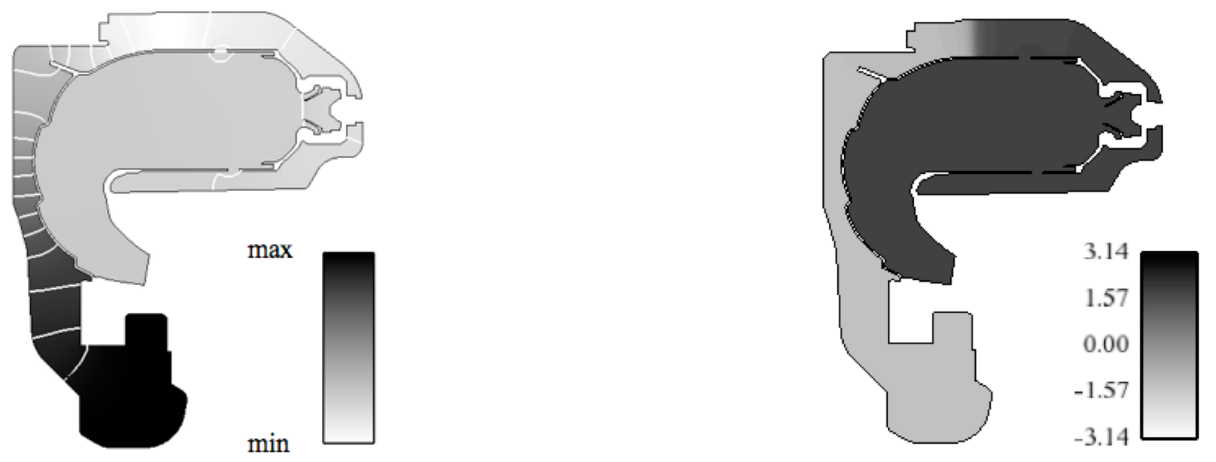

Fig. 17 Field of $|\hat{p}|$ (left) and $\operatorname{Arg}(\hat{p})$ (right) for the $1 L$ mode without plate MP2.

A study of the influence of the bias flow speed in the holes was also conducted in [40]. A bias flow speed $U_{m d}$ inducing a maximum damping was highlighted. The purpose of the next section is to illustrate how an acoustic energy analysis can be used to quantify the effect of each MP on the overall stability of the configuration.

\section{Energy analysis on the second longitudinal mode (2L)}

1. Spatial structure can stand for the efficiency of plates

Since the damping of the mode $2 \mathrm{~L}$ is more significant than the $1 \mathrm{~L}$ mode, an energy budget is now conducted on the second longitudinal mode. It proves to be damped by about $47 \%$. The results are gathered in Table 5. Clearly, plate MP2 is again the most efficient at damping. The distribution of $|\hat{p}|$ and $\operatorname{Arg}(\hat{p})$ is given in Fig. 18. The pressure node is located above MP2, inducing the pressure drop to be maximum across plate MP2, thus inducing a significant damping from this very plate (see Eq. (50)).

Since it is responsible for almost half of the damping, another case is run without plate MP2 to see if it modifies the contribution of the other plates. A null acoustic speed condition is applied in this case on plate MP2. The frequency of the mode is hardly changed. Fig. 18 shows the distribution of the pressure.

As found for the first longitudinal mode, the damping drops (to $27.6 \%$ in this case), which is close to the total damping minus the contribution of plate MP2. This can be explained by the fact that at the actual regime, the presence of the MPs doesn't have a strong impact. The distribution 
of the phase changes. Once again, the contribution of the other plates is hardly changed.

\begin{tabular}{|c|c|c|c|c|c|c|c|c|c|}
\hline $2 \mathrm{~L}$ & $\frac{\bar{E}(t=T)}{\bar{E}(t=0)}-1$ & $R H S$ & Error & $\mathrm{MP} 1$ & $\mathrm{MP} 1 \mathrm{i}$ & $\mathrm{MP} 2$ & $\mathrm{MP} 2 \mathrm{i}$ & $\mathrm{MP} 3$ & $\mathrm{MP} 3 \mathrm{i}$ \\
\hline \hline all MP & -47.1 & -48.2 & 2.46 & $4.6(9.54)$ & $0.68(1.41)$ & $\mathbf{2 2 . 4 6}(\mathbf{4 6 . 6})$ & $3.67(7.614)$ & $10.08(20.9)$ & $6.8(14.1)$ \\
\hline no MP2 & -29 & -28.2 & 2.97 & $5.28(18.7)$ & $0.63(2.23)$ & - & $4.01(14.22)$ & $11.3(40.1)$ & $7.6(27)$ \\
\hline
\end{tabular}

Table 5 Acoustic energy analysis on the second longitudinal mode (2L).
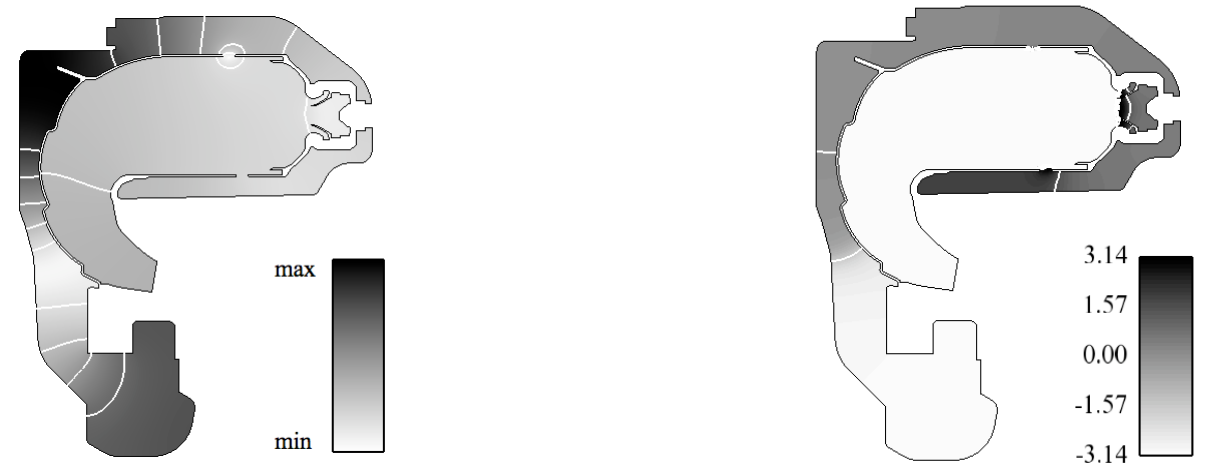

Fig. $18|\hat{p}|$ (left) and $\operatorname{Arg}(\hat{p})$ (right), 2L, all MP.
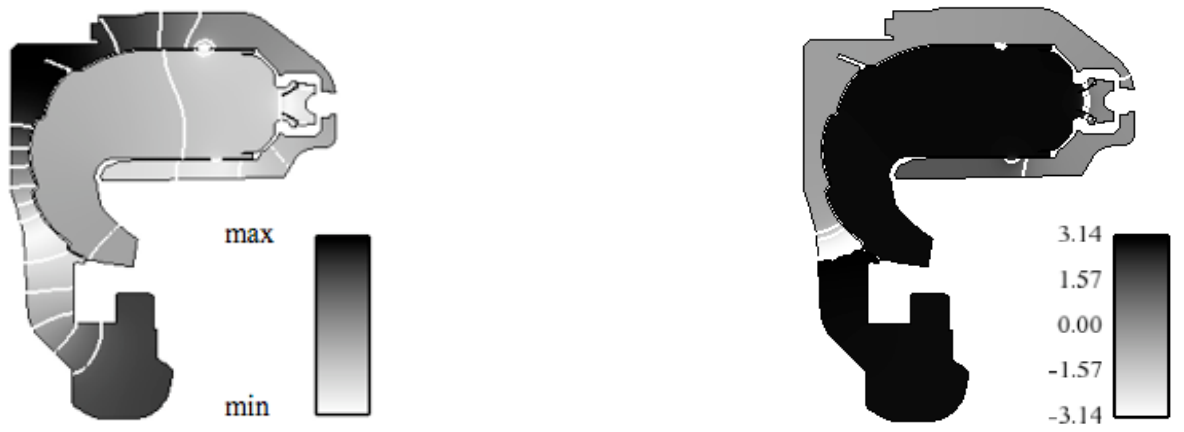

Fig. $19|\hat{p}|$ (left) and $\operatorname{Arg}(\hat{p})$ (right), 2L, no MP2.

D. Energy analysis on the $1 \mathrm{~L}$ and $2 \mathrm{~L}$ modes with maximum damping speed $\left(U=U_{m d}\right)$

As shown in [40], a bias flow speed for which the effect of the MP is significantly larger can be defined. This speed is refered to as $U_{m d}$. The same energy study is run changing the actual speed to $U_{m d}$. For these computations, a uniform speed equal to $U_{m d}$ has been applied on all MPs. Table 6 shows that withdrawing plate MP2 has here a strong impact on the contributions of other plates. 
Plate MP2 is much more efficient. Its damping contribution is up to $82 \%$ of the total damping which has itself increased. Plates MP1, MP3 and MP3i are much more efficient at damping, inducing the whole damping not to be just cut down by the withdrawing of plate MP2. Thus, contrary to the previous case with $U \neq U_{m d}$, the presence of MP2 modifies the mode structure so that the damping generated by the other MPs is changed. In other words, the overall damping is not the sum of all the damping obtained by considering each MP separately.

\begin{tabular}{|c|c|c|c|c|c|c|c|c|}
\hline $1 \mathrm{~L}$ & $e^{2 \omega_{i} T}-1$ & $\epsilon$ & $\mathrm{MP} 1$ & $\mathrm{MP} 1 \mathrm{i}$ & $\mathrm{MP} 2$ & $\mathrm{MP} 2 \mathrm{i}$ & $\mathrm{MP} 3$ & $\mathrm{MP} 3 \mathrm{i}$ \\
\hline \hline$U=U_{\mathrm{md}}$, all MPs & -33.4 & 1.54 & 3.3 & $2.10 .10^{-2}$ & $\mathbf{2 8 . 4}$ & 0.19 & 2.12 & 0.679 \\
\hline & & & 9.51 & 0.06 & $\mathbf{8 1 . 8}$ & 0.55 & 6.11 & 1.96 \\
\hline$U=U_{\mathrm{md}}$, no MP2 & -8.3 & 1.50 & 5.08 & $2.6 .10^{-2}$ & - & $8.6 .10^{-2}$ & 3.6 & 1 \\
\hline & & & 51.9 & 0.266 & - & 0.87 & 36.7 & 10.2 \\
\hline
\end{tabular}

Table 6 Acoustic energy analysis on the second longitudinal mode $(2 \mathrm{~L}), U=U_{m d}$.

Figure 20 shows the mode structure at $U=U_{m d}$ when all MPs are active and Figure 21 shows the case where MP2 is a replaced by an impermeable wall. The pressure jump is maximum across MP2 in Fig. 20 but Fig. 21 shows that the mode structure has changed. The contribution of the plates are therefore all changed. Table 7 shows the same trend of results on the $2 \mathrm{~L}$ mode. Withdrawing plate MP2 has also here a strong impact on the contributions of other plates. Plates MP1, MP3 and MP3i are much more efficient at damping, inducing the whole damping not to be just cut down by the withdrawing of plate MP2.

\begin{tabular}{|c|c|c|c|c|c|c|c|c|c|}
\hline $2 \mathrm{~L}$ & $\frac{\bar{E}(t=T)}{\bar{E}(t=0)}-1$ & $R H S$ & Relative error & MP1 & MP1i & MP2 & MP2i & MP3 & MP3i \\
\hline \hline$U=U_{m d}$, all MP & -73.1 & -74.4 & 2 & $8.62(11.6)$ & $0.17(0.23)$ & $\mathbf{4 6 . 8}(\mathbf{6 2 . 9})$ & $1.2(1.6)$ & $11.6(15.6)$ & $5.96(8)$ \\
\hline$U=U_{m d}$, no MP2 & -44.81 & -46.07 & 2.83 & $15.14(33.8)$ & $0.33(0.73)$ & - & $1.1(2.4)$ & $19.64(43.8)$ & $9.84(21.9)$ \\
\hline
\end{tabular}

Table 7 Acoustic energy analysis on the second longitudinal mode (2L), $U=U_{m d}$.

In complex configurations, the damping contributions of all MPs cannot be simply added. Since the damping effect of a MP depends on the mode structure, its contribution can be significantly 
changed by adding or removing another acousic device even if its parameters are kept the same.

This has strong consequences in terms of acoustic optimization of gas turbines.
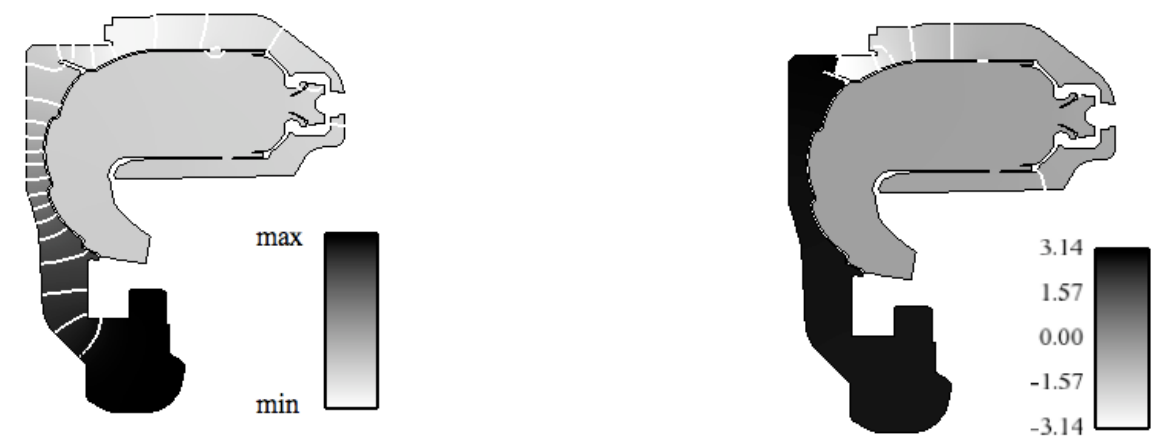

Fig. 20 Field of $|\hat{p}|$ (left) and $\operatorname{Arg}(\hat{p})$ (right) for the $1 L$ mode, $U=U_{\mathrm{md}}$.
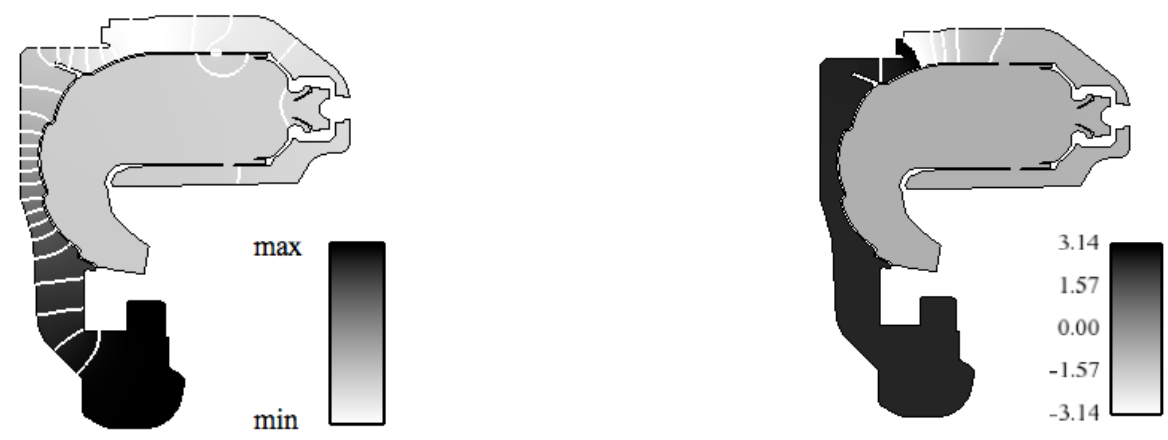

Fig. 21 Field of $|\hat{p}|$ (left) and $\operatorname{Arg}(\hat{p})$ (right), $U=U_{\mathrm{md}}$, without MP2

\section{Computation of the whole chamber: azimuthal modes}

The frequency values of the modes computed with the 360 degree mesh are given in Table 8 . The first mode of the full geometry corresponds to the first longitudinal one already discussed in Section VA. The value of the frequency is close to the one obtained with a one-sector computation and Figure 22 shows that the distributions of $|\hat{p}|$ and $\operatorname{Arg}(\hat{p})$ does not depend of the azimuthal coordinate.

The next two modes correspond to the first azimuthal (1A) and second azimuthal mode (2A) respectively. Each of these modes are double modes in the sense that two different pressure fluctuations correspond to the same frequency of oscillation and damping rate. The modes are called 


\begin{tabular}{|c|c|c|c|c|c|c|}
\hline & \multicolumn{2}{|c|}{$1 \mathrm{~L}$} & \multicolumn{2}{c|}{$1 \mathrm{~A}$} & \multicolumn{2}{c|}{$2 \mathrm{~A}$} \\
\hline \hline & $\operatorname{Re}(f)$ & $\operatorname{Im}(f)$ & $\operatorname{Re}(f)$ & $\operatorname{Im}(f)$ & $\operatorname{Re}(f)$ & $\operatorname{Im}(f)$ \\
\hline & $509.9 \mathrm{~Hz}$ & $-2.54 \mathrm{~Hz}$ & $615.6 \mathrm{~Hz}$ & $-0.92 \mathrm{~Hz}$ & $985 \mathrm{~Hz}$ & $-8.38 \mathrm{~Hz}$ \\
\hline$A_{\text {en }}$ & \multicolumn{2}{|c|}{$6.06 \%$} & \multicolumn{2}{|c|}{$1.86 \%$} & \multicolumn{2}{c|}{$10.1 \%$} \\
\hline
\end{tabular}

Table 8 Frequencies obtained by a 360-degree run (1L, 1A and 2A).
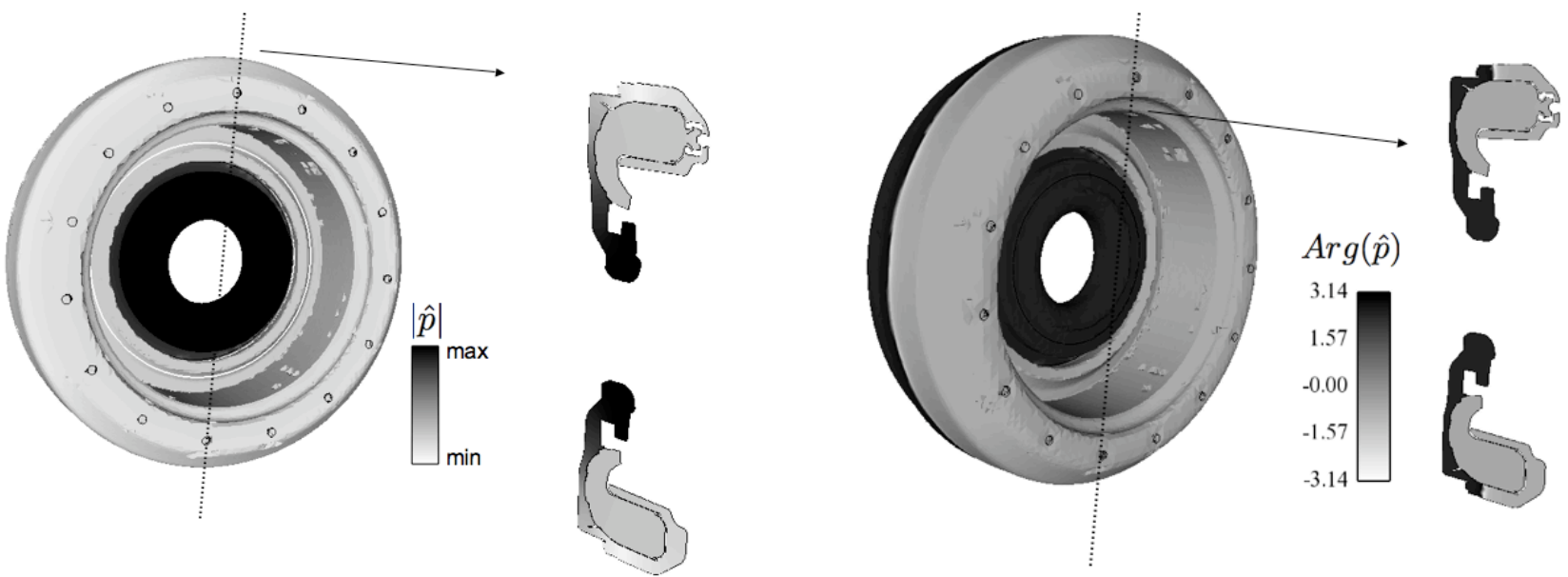

Fig. 22 Field of $|\hat{p}|$ and $\operatorname{Arg}(\hat{p})$ (1L mode). 360-degree mesh.

orthogonal [36, 44, 45]. Figure 23 shows the pressure field of the two modes associated to the frequency of the 1A mode of Table 8 while Fig.24 shows the argument field of these two modes clearly showing their orthogonality. These two pressure fluctuations are close to marginal, indicating that none of the MP is efficient at damping them. On the contrary, the $2 \mathrm{~A}$ mode is clearly damped by the MPs. The pressure and argument fields of the $2 \mathrm{~A}$ mode are shown in Fig. 25 and Fig. 26 respectively. Due to the complexity of the $2 \mathrm{~A}$ mode, two perspectives of the two sides of the annular chamber are shown for one mode. The other $2 \mathrm{~A}$ mode corresponding to the same frequency is simply has a shape simply rotated by 90 degrees. 

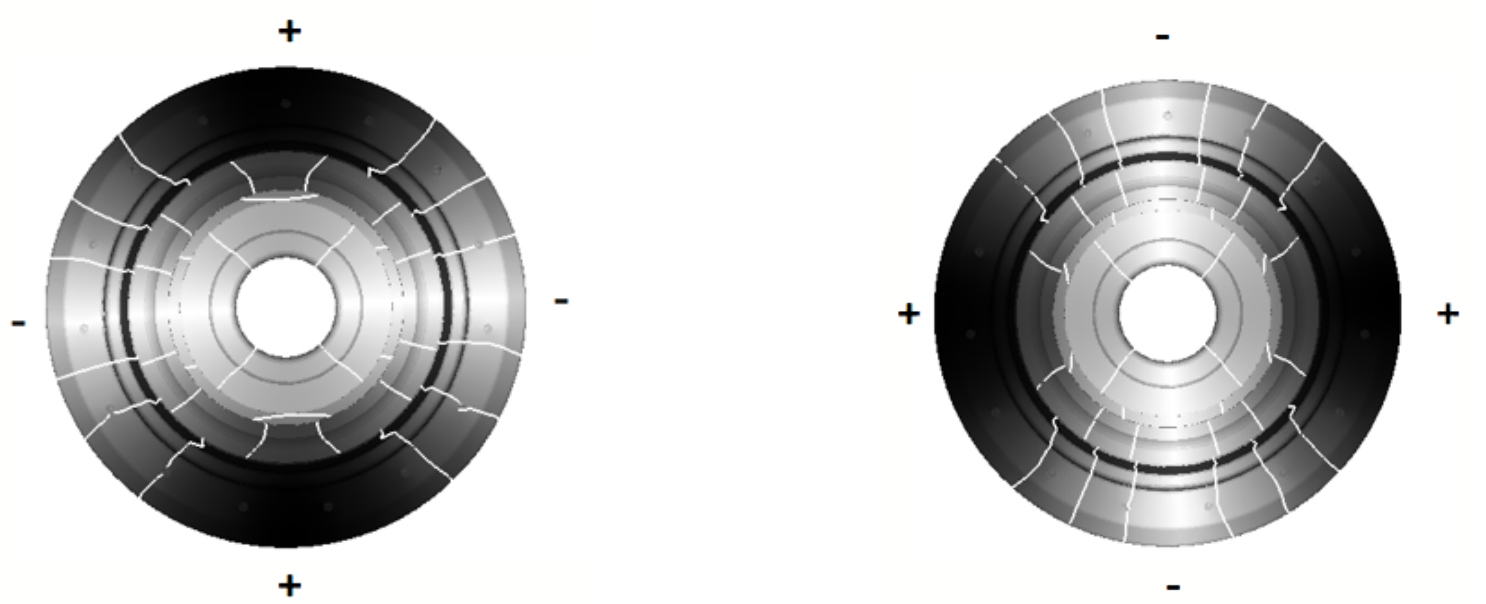

Fig. 23 Field of $|\hat{p}|$ for the $1 A$ mode.
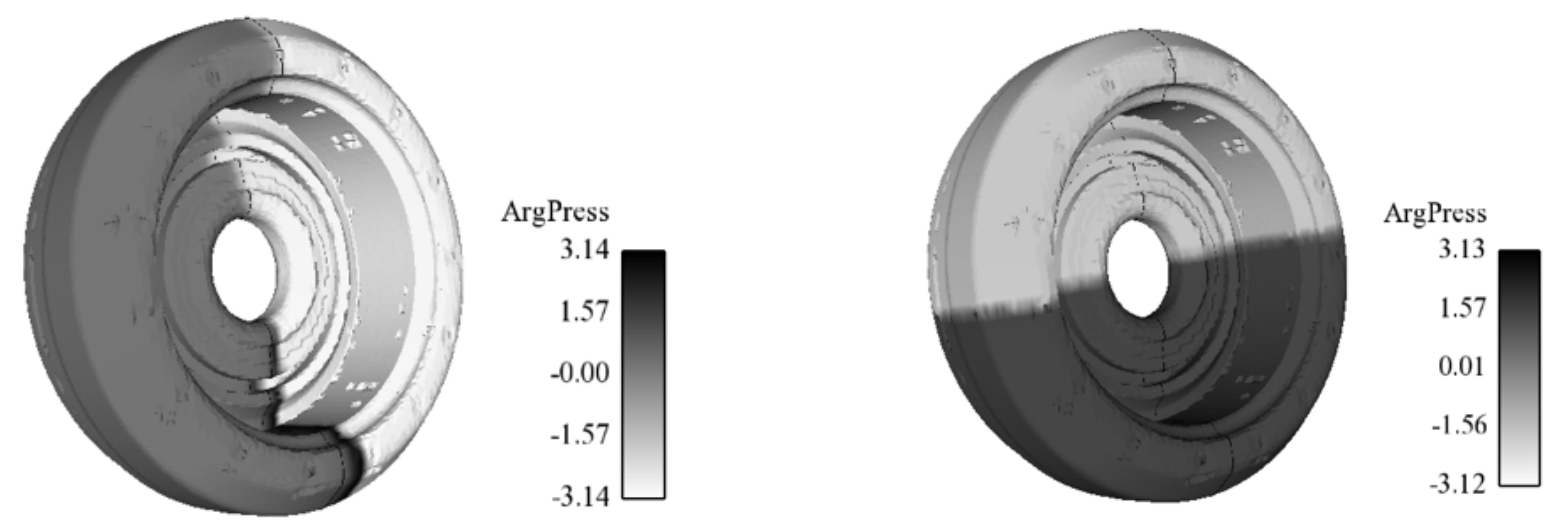

Fig. 24 Field of $\operatorname{Arg}(\hat{p})$ for the $1 A$ mode.
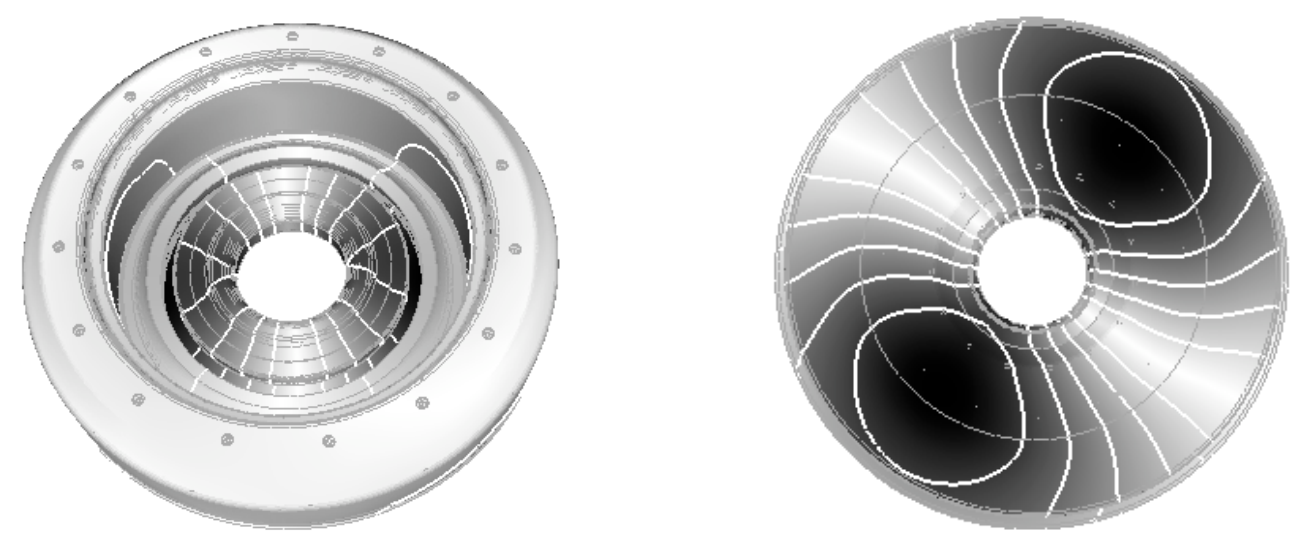

Fig. 25 Field of $|\hat{p}|$ for the $2 A$ mode. View of both sides of the chamber.

\section{Conclusion}

A model for the acoustic behavior of perforated plates was implemented within a general purpose Helmholtz solver. This tool allows to take into account MPs when performing the acoustic analysis 

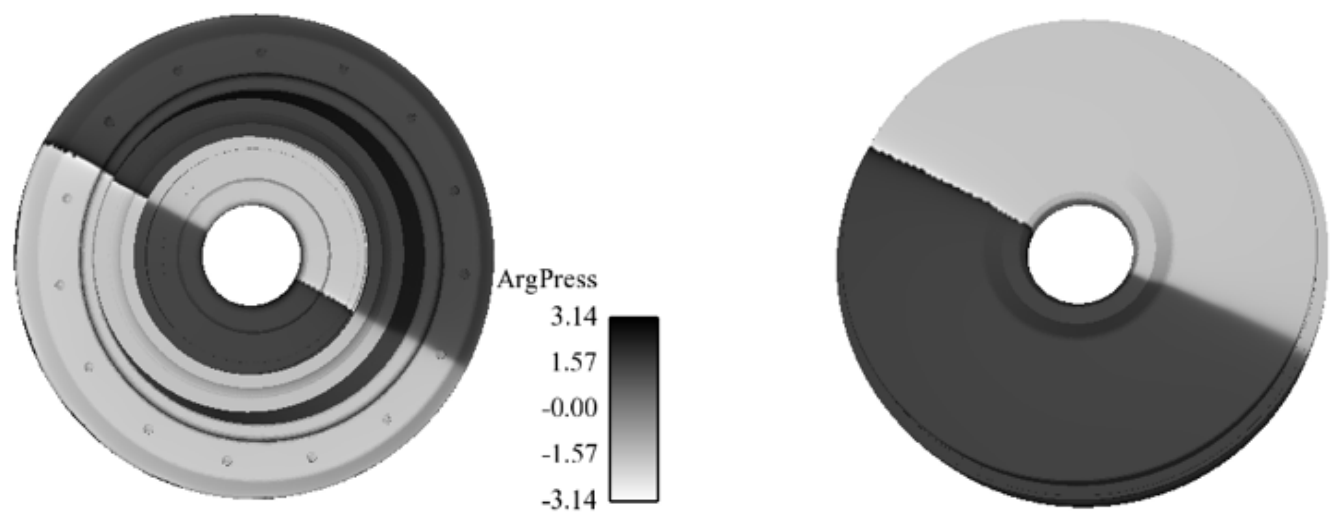

Fig. 26 Field of $\operatorname{Arg}(\hat{p})$ (mode 2A).

of industrial chambers. Besides, the budget of acoustic energy was analyzed for some of the modes computed. This allows to know which perforated plates are the most efficient for damping any acoustic mode and gives some clues why. For the particular industrial case considered, the plate located near the inlet is found to be the most efficient. It is indeed located where the pressure drop is the most important. In cases where the perforated plates do not have a too strong influence, the damping is linear and the total damping is just and only the sum of all the MP contributions. When the plates have a stronger influence and impact on the mode structure, it is illustrated that the effect of a MP area can only be computed by accounting for all the other MPs or significant acoustic devices. This justifies the efforts in developing general purpose Helmholtz solvers for the acoustic design of gas turbines of the future.

\section{Acknowledgments}

The authors are grateful to CINES (Centre Informatique National pour l'Enseignement Supérieur) for the access to computing resources and to SNECMA Moteurs for funding the thesis of the first author. The authors would also like to thank Dr. Simon Mendez for fruitful discussions during the course of this study.

\section{References}

[1] Lieuwen, T. and Yang, V., Combustion Instabilities in Gas Turbine Engines. Operational Experience, Fundamental Mechanisms and Modeling, Progress in Astronautics and Aeronautics, 2005. 
[2] Poinsot, T. and Veynante, D., Theoretical and Numerical Combustion, R.T. Edwards, 2nd edition., 2005.

[3] Manesh, K., Constantinescu, G., Apte, S., Iaccarino, G., Ham, F., and Moin, P., "Large eddy simulation of reacting turbulent flows in complex geometries." ASME J. Appl. Mech., Vol. 73, No. 3, May 2006, pp. $374-381$.

[4] Schmitt, P., Poinsot, T., Schuermans, B., and Geigle, K., "Large-eddy simulation and experimental study of heat transfer, nitric oxide emissions and combustion instability in a swirled turbulent high pressure burner." J. Fluid Mech., Vol. 570, 2007, pp. 17-46.

[5] Moin, P. and Apte, S., "Large-eddy simulation of realistic gas turbine combustors." J. Fluid Mech. , Vol. 44, No. 4, 2006, pp. 698-708.

[6] Selle, L., Lartigue, G., Poinsot, T., Koch, R., Schildmacher, K., Krebs, W., Prade, B., Kaufmann, P., and Veynante, D., "Compressible large-eddy simulation of turbulent combustion in complex geometry on unstructured meshes," Combust. Flame, Vol. 137, No. 4, June 2004, pp. 489-505.

[7] Staffelbach, G., Gicquel, L., Boudier, G., and Poinsot, T., "Large Eddy Simulation of self excited azimuthal modes in annular combustors." Proceedings of the Combustion Institute, Vol. 32, No. 2, 2009, pp. 2909-2916.

[8] Wolf, P., Staffelbach, G., Roux, A., Gicquel, L., Poinsot, T., and Moureau, V., "Massively parallel LES of azimuthal thermo-acoustic instabilities in annular gas turbines," C. R. Acad. Sci. , Vol. 337, No. 6-7, 2009, pp. 385-394.

[9] Giauque, A., Selle, L., Poinsot, T., Buechner, H., Kaufman, P., and Krebs, W., "System identification of a large-scale swirled partially premixed combustor using LES and measurements." J. Turb. , Vol. 6, No. 21, 2005, pp. 1-20.

[10] Noiray, N., Durox, D., Schuller, T., and Candel, S., "A unified framework for nonlinear combustion instability analysis based on the flame describing function," J. Fluid Mech. , Vol. 615, 2008, pp. 139167.

[11] Selle, L., Benoit, L., Poinsot, T., Nicoud, F., and Krebs, W., "Joint use of Compressible Large-Eddy Simulation and Helmoltz solvers for the analysis of rotating modes in an industrial swirled burner." Combust. Flame, Vol. 145, No. 1-2, 2006, pp. 194-205.

[12] Martin, C., Benoit, L., Sommerer, Y., Nicoud, F., and Poinsot, T., "Large-Eddy Simulation and Acoustic Analysis of a Swirled Staged Turbulent Combustor," AIAA Journal , Vol. 44, No. 4, 2006.

[13] Boudier, G., Lamarque, N., Sensiau, C., Staffelbach, G., Poinsot, T., and Moureau, V., "Investigating the thermoacoustic instability of a real gas turbine combustion chamber using Large Eddy Simulation," 
11th CEAS-ASC Workshop and 2nd Scientific Workshop of X3 Noise, Lisbon, Portugal, 2007.

[14] Sensiau, C., Nicoud, F., and Poinsot, T., "Thermoacoustic analysis of an helicopter combustion chamber," 14th AIAA/CEAS Aeroacoustics Conference, Vancouver, 5-7 May 2008.

[15] Sensiau, C., Nicoud, F., and Poinsot, T., "A tool to study azimuthal and spinning modes in annular combustors," International Journal of Aeroacoustics., Vol. 8, No. 1, 2009, pp. 57-68.

[16] Lefebvre, A. H., Gas Turbines Combustion, Taylor \& Francis, 1999.

[17] Auregan, Y. and Pachebat, M., "Measurement of the nonlinear behavior of acoustical rigid porous materials," Phys. Fluids, Vol. 11, No. 6, 1999, pp. 1342-1345.

[18] Cummings, A. and Eversman, W., "High amplitude acoustic transmission through duct terminations: theory," J. Sound Vib. , Vol. 91, No. 4, December 1983, pp. 503-518.

[19] Cummings, A., "Acoustic Nonlinearities and Power Losses at Orifices," AIAA Journal, Vol. 22, No. 6, 1984, pp. 786-792.

[20] Ingard, U. and Ising, H., "Acoustic non linearity of an Orifice," J. Acous. Soc. Am. , Vol. 41, No. 6, 1967, pp. 1582-1583.

[21] Melling, T., "The acoustic impedance of perforates at medium and high sound pressure levels," J. Sound Vib. , Vol. 29, No. 1, 1973, pp. 1-65.

[22] Bechert, D. W., "Sound absorption caused by vorticity shedding, demonstrated with a jet flow," J. Sound Vib. , Vol. 70, No. 3, 1980, pp. 389-405.

[23] Howe, "On the Theory of Unsteady High Reynolds Number Flow Through a Circular Aperture," Proc. Roy. Soc. Lond. A, Vol. 366, No. 1725, June 1979, pp. 205-223.

[24] Hughes, I. and Dowling, A., "The absorption of sound by perforated linings," J. Fluid Mech. , Vol. 218, 1990, pp. 299-335.

[25] Dean, P., "On the "in-situ" control of acoustic liner attenuation," American Society of Mechanical Engineers, Gas Turbine Conference and Products Show, New Orleans, Louisiana, March 21-25 1976, p. 8.

[26] Yang, H., Bao, M., Pakula, L., and French, P., "Damping caused by the gas flow in the holes of perforated structures," Proceedings of the SPIE- The International Society for Optical Engineering, Vol. 4593, 2001, pp. 307-313.

[27] Grace, S. M., Horan, K. P., and Howe, M. S., "The influence of shape on the rayleigh conductivity of a wall aperture in the presence of grazing flow," J. Fluids Struct. , Vol. 12, No. 3, 1998, pp. 335-351.

[28] Tran, N., Ducruix, S., and Schuller, T., "Damping combustion instabilities with perforates at the premixer inlet of a swirled burner," Proc. Combust. Inst., Vol. 32, No. 2, 2009, pp. 2917-2924. 
[29] Tran, N., Ducruix, S., and Schuller, T., "Passive control of the inlet acoustic boundary of a swirled burner at high amplitude combustion instabilities." J. Eng. Gas Turb. and Power, Vol. 131, 2009.

[30] Mendez, S. and Nicoud, F., "Numerical investigation of an anisothermal turbulent flow with effusion," 5th International Symposium on Turbulence and Shear Flow Phenomena, Berlin, Germany, 2007.

[31] Mendez, S. and Nicoud, F., "Adiabatic homogeneous model model for flow around a multiperforated plate," AIAA Journal, Vol. 46, No. 10, 2008, pp. 2623-2633.

[32] Mendez, S. and Eldredge, J., "Acoustic modeling of perforated plates with bias flow for Large-Eddy Simulations," J. Comput. Phys. , Vol. 228, No. 13, LES 2009, pp. 4757-4772.

[33] Jing, X. Sun, X., "Effect of Plate thickness on Impedance of Perforated Plates," AIAA Journal, Vol. 38, No. 9, 2000, pp. 1573-1578.

[34] Sun, X., Jing, X., H, Z., and Shi, Y., "Effect of Grazing-Bias Flow Interaction on Acoustic Impedance of Perforated Plates," J. Sound Vib. , Vol. 254, No. 3, 2002, pp. 557-573.

[35] Howe, M., Scott, M., and Sipcic, S., "The influence of tangential mean flow on the Rayleigh conductivity of an aperture," Proc. Roy. Soc., Vol. 452, 1996, pp. 2303-2317.

[36] Nicoud, F., Benoit, L., and Sensiau, C., "Acoustic Modes in Combustors with Complex Impedances and Multidimensional Active Flames," AIAA Journal, Vol. 45, No. 2, 2007, pp. 426-441.

[37] Lehoucq, R. and Sorensen, D., "Arpack: Solution of large scale eigenvalue problems with implicitly restarted arnoldi methods. www.caam.rice.edu/software/arpack." User's guide, www.caam.rice.edu/software/arpack, 1997.

[38] Rayleigh, L., The Theory of Sound, Mac Millan (reprinted by Dover, New York, 1945), 1894.

[39] Luong, T., Howe, M. S., and McGowan, R. S., "On the Rayleigh conductivity of a bias-flow aperture," J. Fluids Struct. , Vol. 21, No. 8, 2005, pp. 769-778.

[40] Gullaud, E., Mendez, S., Sensiau, C., Nicoud, F., and Poinsot, T., "Effect of multiperforated plates on the acoustic modes in combustors," C. R. Acad. Sci. , Vol. 337, No. 6-7, 2009, pp. 406-414.

[41] Blackstock, D. T., Fundamentals of Physical Acoustics, John Wiley \& Sons, 2000.

[42] Howe, M. S., Acoustics of Fluid-Structure Interaction, Cambridge University Press, 1998.

[43] Boudier, G., Lamarque, N., Staffelbach, G., Gicquel, L. Y. M., and Poinsot, T., "Thermo-acoustic stability of a helicopter gas turbine combustor using large-eddy simulations." International Journal of Aeroacoustics, Vol. 8, No. 1, 2009, pp. 69-94.

[44] Noiray, N., Bothien, M., and Schuermans, B., "Analytical and numerical analysis of staging concepts in annular gas turbines," n3l-Int'l Summer School and Workshop on Non-normal and non linear effects in aero and thermoacoustics., 2010. 
[45] Schuermans, B., Paschereit, C. O., and Monkewitz, P., "Non-linear combustion instabilities in annular gas-turbine combustors," 44th AIAA Aerospace Sciences Meeting and Exhibit, January 9-12, 2006, Reno, NV, USA, 2006. 